\title{
Left-right SU(4) vector leptoquark model for flavor anomalies
}

\author{
Bartosz Fornal, Sri Aditya Gadam, and Benjamín Grinstein \\ Department of Physics, University of California, San Diego, \\ 9500 Gilman Drive, La Jolla, California 92093, USA
}

(Received 12 December 2018; published 18 March 2019)

\begin{abstract}
Building on our recent proposal to explain the experimental hints of new physics in $B$ meson decays within the framework of Pati-Salam quark-lepton unification, through the interactions of the $(3,1)_{2 / 3}$ vector leptoquark, we construct a realistic model of this type based on the gauge group $\mathrm{SU}(4)_{L} \times \mathrm{SU}(4)_{R} \times$ $\mathrm{SU}(2)_{L} \times \mathrm{U}(1)^{\prime}$ and consistent with all experimental constraints. The key feature of the model is that $\mathrm{SU}(4)_{R}$ is broken at a high scale, which suppresses right-handed lepton flavor changing currents at the low scale and evades the stringent bounds from searches for lepton flavor violation. The mass of the leptoquark can be as low as $10 \mathrm{TeV}$ without the need to introduce mixing of quarks or leptons with new vectorlike fermions. We provide a comprehensive list of model-independent bounds from low energy processes on the couplings in the effective Hamiltonian that arises from generic leptoquark interactions, and then apply these to the model presented here. We discuss various meson decay channels that can be used to probe the model and we investigate the prospects for discovering the new gauge boson at future colliders.
\end{abstract}

DOI: 10.1103/PhysRevD.99.055025

\section{INTRODUCTION}

The Standard Model (SM) provides a remarkably successful description of nature at the elementary particle level and, so far, there are only a handful of experimental indications of deviations from its predictions. Perhaps the most significant direct hint of physics beyond the SM are the recently observed anomalies in $B$ meson decays [1,2], which suggest that lepton universality might be violated. Assuming that those anomalies are not a result of experimental systematics, they are best accounted for by the vector leptoquark $(3,1)_{2 / 3}$ or $(3,3)_{2 / 3}[3-5]$. However, building viable UV complete models involving those particles is challenging, especially in light of very stringent constraints on lepton flavor violation (LFV) from various experimental searches.

The first attempt to construct a vector leptoquark model for the $R_{K^{(*)}}$ anomalies was made in [6], where we proposed that the vector leptoquark $(3,1)_{2 / 3}$ explaining the anomalies might be the gauge boson of a theory with Pati-Salam unification. The conclusion was that the minimal model based on $\mathrm{SU}(4) \times \mathrm{SU}(2)_{L} \times \mathrm{SU}(2)_{R}$ is not capable of this because of strict bounds on kaon and $B$ meson rare decays [7-12]. The underlying problem in that model arises from the interference between left-handed (LH) and right-handed

Published by the American Physical Society under the terms of the Creative Commons Attribution 4.0 International license. Further distribution of this work must maintain attribution to the author(s) and the published article's title, journal citation, and DOI. Funded by SCOAP .
(RH) lepton flavor changing currents. We outlined a possible solution to this: extending the gauge group to $\mathrm{SU}(4)_{L} \times \mathrm{SU}(4)_{R} \times \mathrm{SU}(2)_{L} \times \mathrm{U}(1)^{\prime}$ and breaking $\mathrm{SU}(4)_{R}$ at a high scale, such that the RH lepton flavor changing currents are suppressed.

A viable realization of this idea is the subject of this paper. We demonstrate that a Pati-Salam gauge leptoquark as light as $10 \mathrm{TeV}$ can explain the $R_{K^{(*)}}$ anomalies and remain consistent with all experimental bounds without introducing any mixing of quarks and leptons with new fermions. We discuss in detail the constraints arising from LFV searches and show that the absence of RH lepton flavor changing currents relaxes the bounds considerably. The model is expected to have clean signatures at future colliders, which we investigate in the case of the prospective $100 \mathrm{TeV}$ machine.

Several other models for the flavor anomalies based on Pati-Salam unification have been proposed, some appearing almost immediately after our initial work [13-19]. Those models overcome the experimental constraints by mixing all or a subset of SM quarks and leptons with new vectorlike fermions. Other approaches to account for the $B$ meson decay anomalies involving scalar leptoquarks or $Z^{\prime}$ rather than vector leptoquarks have been also proposed (see, e.g., [20-27]).

In Appendix $\mathrm{C}$ we provide a model-independent analysis of the low energy consequences of a $(3,1)_{2 / 3}$ vector leptoquark that interacts with both $\mathrm{LH}$ and $\mathrm{RH}$ fields. We present an extensive list of bounds from flavor physics on generic coupling constants in this model-independent approach; Appendix $\mathrm{C}$ is thus a resource in its own right, of 
use to researchers interested in any specific model of this type. Appendix D is one such example, where we apply the results of Appendix $\mathrm{C}$ to the specific Pati-Salam model constructed in this work. The calculations in Appendix C update and extend previous results [7-12]. For instance, for $B$ decays we use the most recent lattice results for the form factors [28], which weaken the bounds considerably compared to assuming the nonphysical values $f_{+}=f_{0}=1$ adopted previously in the literature.

\section{THE MODEL}

The theory we propose is based on the gauge group

$$
\mathrm{SU}(4)_{L} \times \mathrm{SU}(4)_{R} \times \mathrm{SU}(2)_{L} \times \mathrm{U}(1)^{\prime} .
$$

The crucial feature of the model is that the subgroup $\mathrm{SU}(4)_{R}$ is broken at a much higher scale than $\mathrm{SU}(4)_{L}$, leading to a suppression of RH lepton flavor changing currents.

\section{Fermion particle content}

The matter fields in the model, along with their decomposition into $\mathrm{SU}(3)_{c} \times \mathrm{SU}(2)_{L} \times \mathrm{U}(1)_{Y}$ multiplets, are

$$
\begin{aligned}
& \hat{\Psi}_{L}=(4,1,2,0)=(3,2)_{\frac{1}{6}} \oplus(1,2)_{-\frac{1}{2}}, \\
& \hat{\Psi}_{R}^{u}=\left(1,4,1, \frac{1}{2}\right)=(3,1)_{\frac{2}{3}} \oplus(1,1)_{0}, \\
& \hat{\Psi}_{R}^{d}=\left(1,4,1,-\frac{1}{2}\right)=(3,1)_{-\frac{1}{3}} \oplus(1,1)_{-1}, \\
& \hat{\chi}_{L}=(\overline{4}, 1,2,0)=(\overline{3}, 2)_{-\frac{1}{6}} \oplus(1,2)_{\frac{1}{2}}, \\
& \hat{\chi}_{R}=(1, \overline{4}, 2,0)=(\overline{3}, 2)_{-\frac{1}{6}} \oplus(1,2)_{\frac{1}{2}}
\end{aligned}
$$

for each generation, where $\hat{\Psi}_{L}, \hat{\Psi}_{R}^{u}, \hat{\Psi}_{R}^{d}$ contain the SM fields $Q_{L}, L_{L}, u_{R}, d_{R}, e_{R}$ and a RH neutrino $\nu_{R}$, whereas $\hat{\chi}_{L}$, $\hat{\chi}_{R}$ assure gauge anomaly cancellation and result in two vectorlike pairs of fields $Q_{L}^{\prime}, Q_{R}^{\prime}$ and $L_{L}^{\prime}, L_{R}^{\prime}$ that are heavy and do not mix with SM fermions. This is the minimal fermion content for a consistent theory based on the gauge group (1).

\section{Scalar sector and symmetry breaking}

The Higgs sector contains the scalar representations

$$
\begin{array}{ll}
\hat{\Sigma}_{L}=\left(4,1,1, \frac{1}{2}\right), & \hat{\Sigma}_{R}=\left(1,4,1, \frac{1}{2}\right), \quad \hat{\Sigma}=(\overline{4}, 4,1,0), \\
\hat{H}_{d}=\left(4, \overline{4}, 2, \frac{1}{2}\right), & \hat{H}_{u}=\left(4, \overline{4}, 2,-\frac{1}{2}\right) .
\end{array}
$$

The scalar potential is given in Appendix A. The parameters can be chosen such that the fields $\hat{\Sigma}_{L}, \hat{\Sigma}_{R}$ and $\hat{\Sigma}$ develop the vacuum expectation values (VEVs)

$$
\begin{gathered}
\left\langle\hat{\Sigma}_{L}\right\rangle=\frac{v_{L}}{\sqrt{2}}\left(\begin{array}{l}
0 \\
0 \\
0 \\
1
\end{array}\right), \quad\left\langle\hat{\Sigma}_{R}\right\rangle=\frac{v_{R}}{\sqrt{2}}\left(\begin{array}{l}
0 \\
0 \\
0 \\
1
\end{array}\right), \\
\langle\hat{\Sigma}\rangle=\frac{v_{\Sigma}}{\sqrt{2}}\left(\begin{array}{llll}
1 & 0 & 0 & 0 \\
0 & 1 & 0 & 0 \\
0 & 0 & 1 & 0 \\
0 & 0 & 0 & z
\end{array}\right),
\end{gathered}
$$

where $z>0$. This results in the symmetry breaking pattern

$$
\begin{gathered}
\mathrm{SU}(4)_{L} \times \mathrm{SU}(4)_{R} \times \mathrm{SU}(2)_{L} \times \mathrm{U}(1)^{\prime} \\
\rightarrow \mathrm{SU}(3)_{c} \times \mathrm{SU}(2)_{L} \times \mathrm{U}(1)_{Y}
\end{gathered}
$$

The relation between the SM hypercharge $Y$ and the $\mathrm{U}(1)^{\prime}$ charge $Y^{\prime}$ is given by

$$
Y=Y^{\prime}+\sqrt{\frac{2}{3}}\left(T_{L}^{15}+T_{R}^{15}\right)
$$

where

$$
T_{L}^{15}=T_{R}^{15}=\frac{1}{2 \sqrt{6}} \operatorname{diag}(1,1,1,-3) .
$$

The scalar representations decompose into SM fields as

$$
\begin{aligned}
\hat{\Sigma}_{L} & =(3,1)_{\frac{2}{3}} \oplus(1,1)_{0}, \quad \hat{\Sigma}_{R}=(3,1)_{\frac{2}{3}} \oplus(1,1)_{0}, \\
\hat{\Sigma} & =(8,1)_{0} \oplus(3,1)_{\frac{2}{3}} \oplus(\overline{3}, 1)_{-\frac{2}{3}} \oplus 2(1,1)_{0}, \\
\hat{H}_{d} & =(8,2)_{\frac{1}{2}} \oplus(3,2)_{\frac{7}{6}} \oplus(\overline{3}, 2)_{-\frac{1}{6}} \oplus 2(1,2)_{\frac{1}{2}} \\
& \equiv O_{1} \oplus T_{1} \oplus T_{2}^{\dagger} \oplus S_{1} \oplus S_{2}, \\
\hat{H}_{u} & =(8,2)_{-\frac{1}{2}} \oplus(3,2)_{\frac{1}{6}} \oplus(\overline{3}, 2)_{-\frac{7}{6}} \oplus 2(1,2)_{-\frac{1}{2}} \\
& \equiv O_{2} \oplus T_{3} \oplus T_{4}^{\dagger} \oplus S_{3}^{*} \oplus S_{4}^{*} .
\end{aligned}
$$

Under the symmetry breaking pattern (5) the $\hat{H}_{d}, \hat{H}_{u}$ fields have $(\overline{4}, 4) \rightarrow(\overline{3} \oplus 1) \otimes(3 \oplus 1) ; S_{1}, S_{3}$ stand for the singlets in $1 \otimes 1$, while $S_{2}, S_{4}$ are the singlets in $\overline{3} \otimes 3$. The components of $\hat{\Sigma}_{R}, \hat{\Sigma}_{L}, \hat{\Sigma}$ have masses on the order of the $\mathrm{SU}(4)_{R}$ and $\mathrm{SU}(4)_{L}$ breaking scales. This is also the natural mass scale for the components of $\hat{H}_{d}$ and $\hat{H}_{u}$. However, as shown in Appendix B, it is possible to fine-tune the parameters of the potential such that only one linear combination of the fields $S_{1,2,3,4}$ is light. In particular, there exists a choice of parameters for which the light state is given by

$$
H=-c_{e} S_{1}-c_{d} S_{2}+c_{\nu} S_{3}+c_{u} S_{4},
$$

where $c_{u} \approx 1 \gg c_{d} \gg c_{\nu}$ and $1 \gg c_{e} \gg c_{\nu}$, with the ratio $c_{d}: c_{e} \approx m_{b}: m_{\tau}$. This reduces the scalar sector of the model to that of the SM at low energies. 


\section{Gauge sector}

The gauge and kinetic terms are

$$
\begin{aligned}
\mathcal{L}_{\mathrm{g}+\mathrm{k}}= & -\frac{1}{4} G_{L \mu \nu}^{A} G_{L}^{A \mu \nu}-\frac{1}{4} G_{R \mu \nu}^{A} G_{R}^{A \mu \nu}-\frac{1}{4} W_{\mu \nu}^{a} W^{a \mu \nu} \\
& -\frac{1}{4} Y_{\mu \nu}^{\prime} Y^{\prime \mu \nu}+\left|D_{\mu} \hat{\Sigma}_{L}\right|^{2}+\left|D_{\mu} \hat{\Sigma}_{R}\right|^{2} \\
& +\left|D_{\mu} \hat{\Sigma}\right|^{2}+\left|D_{\mu} \hat{H}_{d}\right|^{2}+\left|D_{\mu} \hat{H}_{u}\right|^{2} \\
& +\overline{\hat{\Psi}}_{L} i \not \hat{\Psi}_{L}+\overline{\hat{\Psi}}_{R}^{u} i \not \hat{\Psi}_{R}^{u}+\overline{\hat{\Psi}}_{R}^{d} i \not \hat{\Psi}_{R}^{d},
\end{aligned}
$$

with $A=1, \ldots, 15$ and $a=1,2,3$. The gauge covariant derivative takes the form

$$
\begin{aligned}
D_{\mu}= & \partial_{\mu}+i g_{L} G_{L \mu}^{A} T_{L}^{A}+i g_{R} G_{R \mu}^{A} T_{R}^{A} \\
& +i g_{2} W_{\mu}^{a} t^{a}+i g_{1}^{\prime} Y_{\mu}^{\prime} Y^{\prime},
\end{aligned}
$$

where $T_{L}^{A}, \quad T_{R}^{A}, \quad t^{a}, \quad Y^{\prime}$ are the $\mathrm{SU}(4)_{L}, \quad \mathrm{SU}(4)_{R}$, $\mathrm{SU}(2)_{L}, \mathrm{U}(1)^{\prime}$ generators. The gauge couplings at the low scale are related to the SM strong and hypercharge couplings via

$$
\begin{aligned}
& g_{s}=\frac{g_{L} g_{R}}{\sqrt{g_{L}^{2}+g_{R}^{2}}}, \\
& g_{1}=\frac{g_{1}^{\prime} g_{L} g_{R}}{\sqrt{\frac{2}{3} g_{1}^{\prime 2}\left(g_{R}^{2}+g_{L}^{2}\right)+g_{L}^{2} g_{R}^{2}}} .
\end{aligned}
$$

The new gauge bosons are

$$
\begin{aligned}
X_{L}=(3,1)_{\frac{2}{3}}, & X_{R}=(3,1)_{\frac{2}{3}}, \quad G^{\prime}=(8,1)_{0}, \\
Z_{L}^{\prime}=(1,1)_{0}, & Z_{R}^{\prime}=(1,1)_{0} .
\end{aligned}
$$

The mass of $G^{\prime}$ is $M_{G^{\prime}}=\frac{1}{\sqrt{2}} \sqrt{g_{L}^{2}+g_{R}^{2}} v_{\Sigma}$. The squared mass matrix for the gauge leptoquarks $X_{L}, X_{R}$ is

$\mathcal{M}_{X}^{2}=\frac{1}{4}\left(\begin{array}{cc}g_{L}^{2}\left[v_{L}^{2}+v_{\Sigma}^{2}\left(1+z^{2}\right)\right] & -2 g_{L} g_{R} v_{\Sigma}^{2} z \\ -2 g_{L} g_{R} v_{\Sigma}^{2} z & g_{R}^{2}\left[v_{R}^{2}+v_{\Sigma}^{2}\left(1+z^{2}\right)\right]\end{array}\right)$.

The leptoquark mass eigenstates can be written as

$$
\left(\begin{array}{l}
X_{1} \\
X_{2}
\end{array}\right)=\left(\begin{array}{cc}
\cos \theta_{4} & \sin \theta_{4} \\
-\sin \theta_{4} & \cos \theta_{4}
\end{array}\right)\left(\begin{array}{l}
X_{L} \\
X_{R}
\end{array}\right),
$$

where the mixing angle $\theta_{4}$ depends on the parameters in Eq. (14). In the limit $v_{R} \gg v_{L}$ and $v_{R} \gg v_{\Sigma}$ the mixing vanishes, $\sin \theta_{4}=0$, and the leptoquark masses become

$$
\begin{aligned}
& M_{X_{1}}=\frac{1}{2} g_{L} \sqrt{v_{L}^{2}+v_{\Sigma}^{2}\left(1+z^{2}\right)}, \\
& M_{X_{2}}=\frac{1}{2} g_{R} v_{R} .
\end{aligned}
$$

The $Z_{L}^{\prime}$ and $Z_{R}^{\prime}$ squared masses are given by the two nonzero eigenvalues of the matrix

$$
\mathcal{M}_{Z^{\prime}}^{2}=\frac{3}{8}\left(\begin{array}{ccc}
g_{L}^{2}\left[v_{L}^{2}+v_{\Sigma}^{2}\left(\frac{1}{3}+z^{2}\right)\right] & -g_{L} g_{R} v_{\Sigma}^{2}\left(\frac{1}{3}+z^{2}\right) & -\frac{\sqrt{2}}{\sqrt{3}} g_{1}^{\prime} g_{L} v_{L}^{2} \\
-g_{L} g_{R} v_{\Sigma}^{2}\left(\frac{1}{3}+z^{2}\right) & g_{R}^{2}\left[v_{R}^{2}+v_{\Sigma}^{2}\left(\frac{1}{3}+z^{2}\right)\right] & -\frac{\sqrt{2}}{\sqrt{3}} g_{1}^{\prime} g_{R} v_{R}^{2} \\
-\frac{\sqrt{2}}{\sqrt{3}} g_{1}^{\prime} g_{L} v_{L}^{2} & -\frac{\sqrt{2}}{\sqrt{3}} g_{1}^{\prime} g_{R} v_{R}^{2} & \frac{2}{3} g_{1}^{\prime 2}\left(v_{L}^{2}+v_{R}^{2}\right)
\end{array}\right) .
$$

Taking the limit $v_{R} \gg v_{L}$ and $v_{R} \gg v_{\Sigma}$ yields

$$
M_{Z_{L}^{\prime}}=\sqrt{\frac{g_{1}^{\prime 2}\left(g_{L}^{2}+g_{R}^{2}\right)+\frac{3}{2} g_{L}^{2} g_{R}^{2}}{8\left(g_{1}^{\prime 2}+\frac{3}{2} g_{R}^{2}\right)}} \sqrt{3 v_{L}^{2}+v_{\Sigma}^{2}\left(1+3 z^{2}\right)}, \quad M_{Z_{R}^{\prime}}=\frac{1}{2} \sqrt{g_{1}^{\prime 2}+\frac{3}{2} g_{R}^{2}} v_{R} .
$$

\section{Fermion masses}

The Yukawa interactions are

$$
\begin{aligned}
\mathcal{L}_{Y} & =y_{i j}^{d} \overline{\hat{\Psi}_{L}^{i}} \hat{H}_{d} \hat{\Psi}_{R}^{d j}+y_{i j}^{u} \overline{\hat{\Psi}_{L}^{i}} \hat{H}_{u} \hat{\Psi}_{R}^{u j}+Y_{i j} \overline{\hat{\chi}_{L}^{i}} \hat{\Sigma} \hat{\chi}_{R}^{j}+\text { H.c. } \\
& \supset y_{i j}^{d} \overline{L_{L}^{i}} S_{1} e_{R}^{j}+y_{i j}^{d} \overline{Q_{L}^{i}} S_{2} d_{R}^{j}+y_{i j}^{u} \overline{L_{L}^{i}} S_{3}^{*} \nu_{R}^{j}+y_{i j}^{u} \overline{Q_{L}^{i}} S_{4}^{*} u_{R}^{j}+\frac{1}{\sqrt{2}} Y_{i j} v_{\Sigma}\left(\overline{Q_{L}^{\prime i}} Q_{R}^{\prime j}+z \overline{L_{L}^{\prime i}} L_{R}^{\prime j}\right)+\text { H.c. } \\
& \supset-c_{e} y_{i j}^{d} \overline{L_{L}^{i}} H e_{R}^{j}-c_{d} y_{i j}^{d} \overline{Q_{L}^{i}} H d_{R}^{j}+c_{\nu} y_{i j}^{u} \overline{L_{L}^{i}} \tilde{H} \nu_{R}^{j}+c_{u} y_{i j}^{u} \overline{Q_{L}^{i}} \tilde{H} u_{R}^{j}+\frac{1}{\sqrt{2}} Y_{i j} v_{\Sigma}\left(\overline{Q_{L}^{\prime i}} Q_{R}^{j j}+z \overline{L_{L}^{\prime i}} L_{R}^{\prime j}\right)+\text { H.c. },
\end{aligned}
$$

where $i, j=1,2,3$ are family indices and the coefficients " $c$ " are those in Eq. (9). Typically, in theories with quark-lepton unification, the up-type quark and neutrino masses of a given generation are the same at the unification scale, and similarly 
the down-type quark and charged lepton masses. In our model this is not the case, but since there are only two Yukawa matrices $y^{u}$ and $y^{d}$, without additional mass contributions the hierarchy of the up-type quark masses is, a priori, the same as for the neutrinos, and the downtype quark mass hierarchy the same as for the charged leptons at the unification scale.

Regarding the up-type quarks and neutrinos, for which the experimentally determined mass hierarchies differ considerably, this is solved by introducing a new scalar representation $\hat{\Phi}_{10}=(1, \overline{10}, 1,-1)$. If the $\mathrm{SM}$ singlet component of $\hat{\Phi}_{10}$ develops a VEV $v_{10}$ at a high scale, this provides a seesaw mechanism for the neutrinos via the interaction

$$
y_{i j}^{u \prime} \overline{\left(\hat{\Psi}_{R}^{u i}\right)^{c}} \hat{\Phi}_{10} \hat{\Psi}_{R}^{u j} .
$$

The contribution to the up-type quarks vanishes. Therefore, the up-type quark masses are $m_{u} \sim y^{u} v$, whereas the neutrino masses are $m_{\nu} \sim\left(c_{\nu} y^{u} v\right)^{2} /\left(y^{u \prime} v_{10}\right)$.

The relative mass hierarchies of the down-type quarks versus charged leptons are not in vast disagreement with experiment. The running of the masses will largely account for $m_{b} / m_{\tau}$. One can also introduce the scalar representation $\hat{\Phi}_{15}=(15,1,1,0)$ into the model, with the SM singlet component developing the $\operatorname{VEV} v_{15} \operatorname{diag}(1,1,1,-3)$. New mass contributions to the down-type quarks and charged leptons would then result from loop processes, parametrized via the effective dimension five interaction $y_{i j}^{d /} \hat{\Psi}_{L}^{i} \hat{H}_{d} \hat{\Psi}_{R}^{d j} \hat{\Phi}_{15} / \Lambda$, and mediated, e.g., by heavy vectorlike fermions, leading to additional mass splitting.

\section{Flavor structure}

In terms of SM fermion mass eigenstates, the interactions of the vector leptoquarks with quarks and leptons are given by

$$
\begin{aligned}
\mathcal{L} \supset & \frac{g_{L}}{\sqrt{2}} X_{L \mu}\left[L_{i j}^{u}\left(\bar{u}^{i} \gamma^{\mu} P_{L} \nu^{j}\right)+L_{i j}^{d}\left(\bar{d}^{i} \gamma^{\mu} P_{L} e^{j}\right)\right] \\
& +\frac{g_{R}}{\sqrt{2}} X_{R \mu}\left[R_{i j}^{u}\left(\bar{u}^{i} \gamma^{\mu} P_{R} \nu^{j}\right)+R_{i j}^{d}\left(\bar{d}^{i} \gamma^{\mu} P_{R} e^{j}\right)\right]+\text { H.c. },
\end{aligned}
$$

where $L^{u / d}, R^{u / d}$ are unitary mixing matrices. They are related via $L^{u}=V L^{d} U$ and $R^{u}=V R^{d} U$, where $V$ is the Cabibbo-Kobayashi-Maskawa matrix and $U$ is the Pontecorvo-Maki-Nakagawa-Sakata matrix.

\section{Proton stability}

The vector boson $(3,1)_{2 / 3}$ does not mediate proton decay [6] and neither do any of the scalars in our model. In particular, for the scalar $(3,2)_{1 / 6}$, which by itself would be problematic [29], gauge invariance forbids tree-level proton decay. In broader terms, the Lagrangian in Eq. (19) is invariant under the global symmetries $\mathrm{U}(1)_{B}^{\prime}$ and $\mathrm{U}(1)_{L}^{\prime}$, with the matter fields $\Psi_{L}, \Psi_{R}^{d}$ and $\Psi_{R}^{u}$ carrying charges $B^{\prime}=L^{\prime}=1 / 4$ and all scalar fields being neutral. After symmetry breaking the charges under the remaining global $\mathrm{U}(1)_{B}$ and $\mathrm{U}(1)_{L}$ are

$$
\begin{aligned}
& B=B^{\prime}+\frac{1}{\sqrt{6}}\left(T_{L}^{15}+T_{R}^{15}\right), \\
& L=L^{\prime}-\frac{\sqrt{6}}{2}\left(T_{L}^{15}+T_{R}^{15}\right),
\end{aligned}
$$

which are simply the SM baryon and lepton number. Proton decay is thus forbidden at all orders in perturbation theory.

\section{FLAVOR ANOMALIES}

In this section we discuss how the vector leptoquark of $\mathrm{SU}(4)_{L}$ can explain the recent hints of physics beyond the $\mathrm{SM}$ in $B$ meson decays, i.e., the deficit in the ratios

$$
\begin{array}{r}
R_{K}=\frac{\operatorname{Br}\left(B^{+} \rightarrow K^{+} \mu^{+} \mu^{-}\right)}{\operatorname{Br}\left(B^{+} \rightarrow K^{+} e^{+} e^{-}\right)}, \\
R_{K^{*}}=\frac{\operatorname{Br}\left(B^{0} \rightarrow K^{* 0} \mu^{+} \mu^{-}\right)}{\operatorname{Br}\left(B^{0} \rightarrow K^{* 0} e^{+} e^{-}\right)}
\end{array}
$$

with respect to SM predictions [1,2]. For an analysis of the anomalies at the effective operator level see [30-37].

To describe the decays in Eq. (23) quantitatively, it is convenient to start out from the effective Lagrangian for flavor changing neutral current processes with a $b \rightarrow s$ transition. Up to four-quark operators, it can be written as

$$
\begin{aligned}
\mathcal{L}= & \frac{4 G_{F}}{\sqrt{2}} V_{t b} V_{t s}^{*} \sum_{i, j}\left[\sum_{k=7}^{10} C_{k}^{i j} \mathcal{O}_{k}^{i j(\prime)}+C_{\nu}^{i j} \mathcal{O}_{\nu}^{i j}\right. \\
& \left.+C_{S}^{i j(\prime)} \mathcal{O}_{S}^{i j(\prime)}+C_{P}^{i j(\prime)} \mathcal{O}_{P}^{i j(\prime)}\right] .
\end{aligned}
$$

The operators $\mathcal{O}_{7}^{i j}$ and $\mathcal{O}_{8}^{i j}$ correspond to electromagnetic and chromomagnetic moment transitions; the $\mathcal{O}_{9}^{i j(\prime)}, \mathcal{O}_{10}^{i j(\prime)}$, $\mathcal{O}_{\nu}^{k l}$ are the semileptonic operators

$$
\begin{aligned}
\mathcal{O}_{9(10)}^{i j} & =\frac{e^{2}}{16 \pi^{2}}\left(\bar{s} \gamma_{\mu} P_{L} b\right)\left[\bar{l}^{i} \gamma^{\mu}\left(\gamma_{5}\right) l^{j}\right], \\
\mathcal{O}_{9(10)}^{i j \prime} & =\frac{e^{2}}{16 \pi^{2}}\left(\bar{s} \gamma_{\mu} P_{R} b\right)\left[\bar{l}^{i} \gamma^{\mu}\left(\gamma_{5}\right) l^{j}\right], \\
\mathcal{O}_{\nu}^{i j} & =\frac{e^{2}}{8 \pi^{2}}\left(\bar{s} \gamma_{\mu} P_{L} b\right)\left(\bar{\nu}^{i} \gamma^{\mu} P_{L} \nu^{j}\right) ;
\end{aligned}
$$

and $\mathcal{O}_{S}^{i j(\prime)}, \mathcal{O}_{P}^{i j(\prime)}$ are the scalar operators 


$$
\begin{aligned}
\mathcal{O}_{S}^{i j(\prime)} & =\frac{e^{2}}{16 \pi^{2}}\left[\bar{s} P_{R(L)} b\right]\left(\bar{l}^{i} l^{j}\right), \\
\mathcal{O}_{P}^{i j(\prime)} & =\frac{e^{2}}{16 \pi^{2}}\left[\bar{s} P_{R(L)} b\right]\left(\bar{l}^{i} \gamma_{5} l^{j}\right) .
\end{aligned}
$$

Tensor operators were neglected since they cannot arise from short-distance new physics with SM linearly realized [4].

Global fits to the $R_{K^{(*)}}$ anomalies and other $b \rightarrow s \ell \ell$ data have been performed [30-37]. These analyses yield similar best fit values for the Wilson coefficients. In what follows we adopt the results of [30], i.e.,

$\operatorname{Re}\left(\Delta C_{9}^{\mu \mu}-\Delta C_{9}^{e e}\right)=-\operatorname{Re}\left(\Delta C_{10}^{\mu \mu}-\Delta C_{10}^{e e}\right) \approx-0.6$,

with the contributions to the remaining Wilson coefficients being small. In our model, the vector leptoquarks $X_{1}, X_{2}$ modify the coefficients by

$$
\begin{aligned}
\Delta C_{9}^{i j} & =-\Delta C_{10}^{i j}=-\frac{\sqrt{2} \pi^{2} g_{L}^{2} L_{2 i}^{d} L_{3 j}^{d *}}{G_{F} e^{2} V_{t b} V_{t s}^{*}}\left[\frac{\cos ^{2} \theta_{4}}{M_{X_{1}}^{2}}+\frac{\sin ^{2} \theta_{4}}{M_{X_{2}}^{2}}\right] \\
\Delta C_{9}^{i j \prime} & =\Delta C_{10}^{i j \prime}=-\frac{\sqrt{2} \pi^{2} g_{R}^{2} R_{2 i}^{d} R_{3 j}^{d *}}{G_{F} e^{2} V_{t b} V_{t s}^{*}}\left[\frac{\sin ^{2} \theta_{4}}{M_{X_{1}}^{2}}+\frac{\cos ^{2} \theta_{4}}{M_{X_{2}}^{2}}\right] \\
\Delta C_{S}^{i j} & =-\Delta C_{P}^{i j} \\
& =-\frac{\sqrt{2} \pi^{2} g_{L} g_{R} R_{2 i}^{d} L_{3 j}^{d *} \sin 2 \theta_{4}}{G_{F} e^{2} V_{t b} V_{t s}^{*}}\left[\frac{1}{M_{X_{1}}^{2}}-\frac{1}{M_{X_{2}}^{2}}\right] \\
\Delta C_{S}^{i j \prime} & =\Delta C_{P}^{i j \prime} \\
& =-\frac{\sqrt{2} \pi^{2} g_{L} g_{R} L_{2 i}^{d} R_{3 j}^{d *} \sin 2 \theta_{4}}{G_{F} e^{2} V_{t b} V_{t s}^{*}}\left[\frac{1}{M_{X_{1}}^{2}}-\frac{1}{M_{X_{2}}^{2}}\right] \\
\Delta C_{\nu}^{i j} & =0 .
\end{aligned}
$$

Guided by the tightness of the bounds from LFV searches (discussed in Sec. IV and Appendix C), we assume that $\mathrm{SU}(4)_{R}$ is broken at a much higher scale than $\mathrm{SU}(4)_{L}$, i.e.,

$$
v_{R} \gg v_{L} \quad \text { and } \quad v_{R} \gg v_{\Sigma} .
$$

This suppresses RH lepton flavor changing currents and results in the contributions to the Wilson coefficients other than $\Delta C_{9,10}^{i j}$ being small. The condition in Eq. (27) becomes

$$
\frac{M_{X_{L}}}{g_{L} \sqrt{\operatorname{Re}\left(L_{22}^{d} L_{32}^{d *}-L_{21}^{d} L_{31}^{d *}\right)}} \approx 23 \mathrm{TeV} .
$$

\section{EXPERIMENTAL CONSTRAINTS}

The leptoquark masses and the mixing matrices are subject to experimental constraints from a number of null searches for LFV, with the most stringent bounds coming from rare decays of pions [38-40], kaons [41-46], $B$ mesons [47-54], $\tau$ leptons [55-58] and $\mu-e$ conversion [59].

Implications of those constraints for Pati-Salam unification have been considered in the literature [7-12], but focused on models in which the vector leptoquark $(3,1)_{2 / 3}$ couples to both $\mathrm{LH}$ and $\mathrm{RH}$ fermion fields with similar strength. The conclusion of those analyses, updated with the most recent experimental bounds [52-54], is that the leptoquark mass has to be $\gtrsim 90 \mathrm{TeV}$ [12]. In addition, constraints from searches for $\mu \rightarrow e \gamma$ when both LH and RH leptoquark interactions are present can push this limit much higher due to the bottom quark mass enhancement of the one-loop diagram (see Appendix $\mathrm{C}$ and also [60] for a discussion of a similar effect in scalar leptoquark models). Such a heavy leptoquark would not explain the $R_{K^{(*)}}$ anomalies, since the required relation, analogous to the one in Eq. (30), could not be satisfied for a perturbative gauge coupling and unitary mixing matrices.

In our model, for a sufficiently high scale of $\mathrm{SU}(4)_{R}$ breaking, the constraints arising from the presence of leptoquark RH couplings to fermions are eliminated and the remaining bounds on LH interactions can be satisfied for a significantly lower leptoquark mass. The tightest limits are listed in the Appendix, for arbitrary LH and RH leptoquark interactions in Appendix $\mathrm{C}$ and for the case of just LH interactions in Appendix D.

If the mixing matrix entries $L_{11}^{d}, L_{12}^{d}$ are $\mathcal{O}(1)$, the limits from searches for $K_{L}^{0} \rightarrow e^{ \pm} \mu^{\mp}$ and $\mu-e$ conversion a priori push the leptoquark mass up to hundreds of $\mathrm{TeV}$ in our model (thousands of $\mathrm{TeV}$ for models in which both $\mathrm{LH}$ and RH leptoquark interactions are present, due to the enhancement of the scalar current contribution; see Appendix C). The bounds, however, are satisfied for a much lighter leptoquark provided $L_{11}^{d}, L_{12}^{d} \ll 1$. Unitarity then implies that $L_{13}^{d} \approx 1$ and $L_{23}^{d}, L_{33}^{d} \ll 1$; therefore $L^{d}$ takes the form

$$
L^{d} \approx e^{i \phi}\left(\begin{array}{ccc}
\delta_{1} & \delta_{2} & 1 \\
e^{i \phi_{1}} \cos \theta & e^{i \phi_{2}} \sin \theta & \delta_{3} \\
-e^{-i \phi_{2}} \sin \theta & e^{-i \phi_{1}} \cos \theta & \delta_{4}
\end{array}\right),
$$

where $\left|\delta_{i}\right| \ll 1$. Note that the suppression of RH flavor changing currents in our model implies that there are no significant bounds from $\pi^{0} \rightarrow \nu \bar{\nu}$ or $K_{L}^{0} \rightarrow \nu \bar{\nu}$.

The remaining entries of $L^{d}$ are subject to further constraints, mainly from $B$ meson and $\tau$ decays. If both $\mathrm{LH}$ and RH leptoquark interactions were present, the $B^{0} \rightarrow$ $\mu^{+} \mu^{-}$decay would provide the most stringent bound. However, with only LH interactions the tightest limits arise from searches for $B^{+} \rightarrow K^{+} e^{ \pm} \mu^{\mp}$. We calculated the corresponding branching fractions (see Appendix C) using the most recent lattice results for the form factors [28] based on the Bourrely-Caprini-Lellouch parametrization [61], 
which relaxes the bounds considerably compared to taking the nonphysical values $f_{+}=f_{0}=1$ [11].

The resulting bound on $M_{X_{L}}$ is minimized for $\theta \approx \pi / 4$ and requires merely $M_{X_{L}} / g_{L} \gtrsim 9.2 \mathrm{TeV}$. Given the relation between the gauge couplings in Eq. (12) and assuming $g_{R} \approx$ $\sqrt{3 \pi}$ (close to the perturbative limit) implies $g_{L} \approx 1.06 g_{s}$, where $g_{s} \approx 0.96$ is the strong coupling constant at $10 \mathrm{TeV}$. This leads to the constraint

$$
M_{X_{L}} \gtrsim 10 \mathrm{TeV} .
$$

(If one chose instead $g_{L}=g_{R}=\sqrt{2} g_{s}$, this would result in the constraint $M_{X_{L}} \gtrsim 14 \mathrm{TeV}$.) Saturating the bound in Eq. (32), the condition in Eq. (30) for explaining the $R_{K^{(*)}}$ anomalies is fulfilled if $\cos \left(\phi_{1}+\phi_{2}\right) \approx 0.18$. We also note that for $M_{X_{L}} \approx 10 \mathrm{TeV}$ one could have $\left|\delta_{i}\right| \sim 0.02$, so the matrix $L^{d}$ in Eq. (31) does not need to be highly tuned.

Finally, let us note that all loop-level constraints, including $K-\bar{K}, B-\bar{B}, B_{s}-\bar{B}_{s}$ mixing; radiative decays $\mu \rightarrow e \gamma$ (see Appendix C), $\tau \rightarrow e \gamma$; anomalous magnetic and electric moments of leptons; $Z \rightarrow b \bar{b}$ and others [62], are satisfied due to the unitarity of $L^{d}$ and a leptoquark mass in excess of $10 \mathrm{TeV}$.

\section{COLLIDER PHENOMENOLOGY}

The aim of this limited phenomenological analysis is to simply demonstrate that the leptoquark $X_{L}$ in our model accounting for the flavor anomalies can be searched for at the next generation collider. Focusing on the proposed $100 \mathrm{TeV}$ Future Circular Collider (FCC), we find that one of the best signatures to look for is provided by the single leptoquark production process

$$
p p \rightarrow X_{L} j \mu^{-} \rightarrow j j \mu^{+} \mu^{-} .
$$

In an in-depth analysis one could also investigate final states involving other leptons, which for the case of neutrinos would lead to missing energy signatures. Pair production of $10 \mathrm{TeV}$ leptoquarks is suppressed even at a $100 \mathrm{TeV}$ collider.

To simulate the SM background and the leptoquark signal for the process (33) we used MadGraph 5 [63] (version 2.6.3) with the default cuts apart from the lower cut on the transverse momentum of jets and leptons, which was set to $300 \mathrm{GeV}$. The leptoquark model file for MadGraph was implemented using FeynRules [64] (version 2.3.32).

Figure 1 plots the number of background $(B)$ and signal $(S)$ events for a leptoquark mass 10, 12 and $14 \mathrm{TeV}$ expected within the first year of FCC running (estimated to be $250 \mathrm{fb}^{-1}$ of data [65]) as a function of the invariant mass of the highest transverse momentum jet $j$ and $\mu^{+}$. Implementing the invariant mass cut $\left|M_{j \mu^{+}}-M_{X_{L}}\right|<\Gamma_{X}$, where $\Gamma_{X}$ is the width of the leptoquark, the significance of

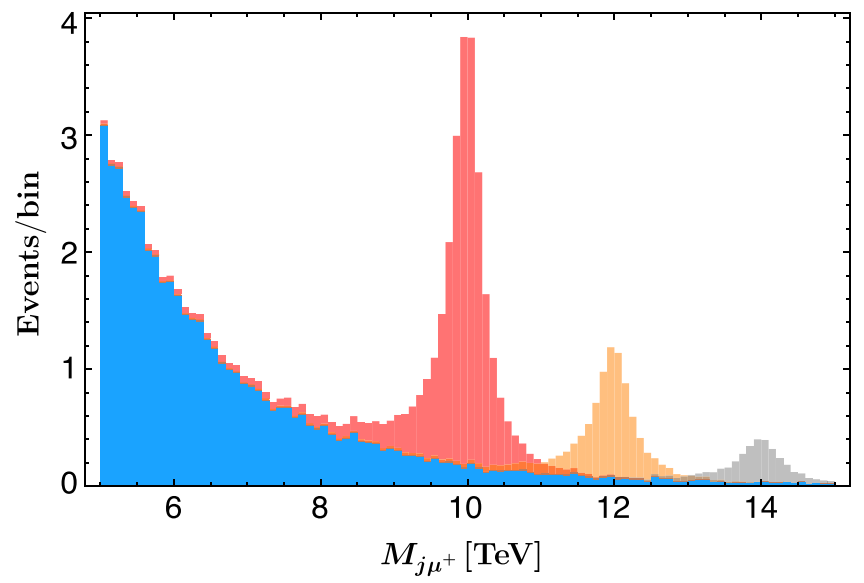

FIG. 1. Expected number of events in $250 \mathrm{fb}^{-1}$ of data collected by a $100 \mathrm{TeV} p p$ collider for the SM background $p p \rightarrow$ $j j \mu^{+} \mu^{-}$(blue) and the leptoquark signal $p p \rightarrow X_{L} j \mu^{-} \rightarrow j j \mu^{+} \mu^{-}$ for masses $M_{X_{L}}=10,12,14 \mathrm{TeV}$ (red, orange, gray) as a function of the invariant mass of the highest $p_{T}$ jet and $\mu^{+}$. The values of parameters discussed in Sec. IV were used.

the signal, $S / \sqrt{B}$, is very high: $19 \sigma$ for $M_{X_{L}}=10 \mathrm{TeV}$, $6.7 \sigma$ for $12 \mathrm{TeV}$ and $4.5 \sigma$ for $14 \mathrm{TeV}$. More sophisticated cuts may make the search more efficient. A detailed analysis of the $X_{L}$ vector leptoquark collider phenomenology is beyond the scope of this paper.

Were the $B$ decay anomalies in $R_{K}$ and $R_{K^{*}}$ confirmed and established, inspection of Eq. (30) indicates that this model could be ruled out at a future $100 \mathrm{TeV}$ high luminosity hadron collider. Not only does the right-hand side of Eq. (30) provide an upper bound on the mass of the vector leptoquark, but Eq. (12) shows that the strength of the coupling constant $g_{L}$ is bounded from below, and therefore the height of the resonant signal in Fig. 1 is bounded from below.

\section{CONCLUSIONS}

We have constructed a new model to account for the recently observed anomalies in $B$ meson decays set within the framework of Pati-Salam unification. The theory avoids all experimental bounds without introducing any vectorlike fields mixing with the Standard Model fermions. This was achieved by suppressing the leptoquark right-handed interactions by associating them with a symmetry broken at a high scale, which eliminates the most stringent constraints arising from the simultaneous presence of left- and right-handed lepton flavor changing currents. In some regions of parameter space the mass of the leptoquark can be as low as $10 \mathrm{TeV}$ while remaining consistent with all experimental data.

The tightest constraints on the model come from the experimental limits on rare kaon, $B$ meson and $\tau$ decays, as well as $\mu-e$ conversion. In the appendix we presented general model-independent formulas for the various decay rates and listed the corresponding bounds. Those results can be used to read off the constraints on any model with one or more $(3,1)_{2 / 3}$ vector leptoquarks with arbitrary 
left- and right-handed interactions with Standard Model quarks and leptons.

In our analysis we chose parameters to explain the $R_{K^{(*)}}$ flavor anomalies. As shown in [3], phenomenological models of the vector leptoquark $(3,1)_{2 / 3}$ can also account for $R_{D^{(*)}}$ anomalies [66-68] (see [69] for an updated fit). The vector leptoquark $(3,1)_{2 / 3}$ in our model is too heavy to account also for the $R_{D^{(*)}}$ anomalies. Still, it has been shown [70] that the scalar leptoquark $(3,2)_{1 / 6}$ might be a good candidate for that. This leptoquark appears in the scalar sector of our model and can be made sufficiently light. It would be interesting to investigate this in more detail.
Currently, there exist many models that account for the hints of lepton universality violation in $B$ meson decays. If these anomalies are established, new physics must emerge at a scale similar to that of the mass of the "left-handed" leptoquark in our model. We have demonstrated that simple kinematic cuts can isolate clearly observable signals with $250 \mathrm{fb}^{-1}$ of accumulated data at a $100 \mathrm{TeV} p p$ collider. Further analysis is badly required to determine whether such an apparatus could distinguish among the many proposed models.

\section{ACKNOWLEDGMENTS}

This research was supported in part by the U.S. Department of Energy Grant No. DE-SC0009919.

\section{APPENDIX A: $\mathrm{SU}(4)_{L} \times \mathrm{SU}(4)_{R}$ SYMMETRY BREAKING}

The scalar potential of the model is given by

$$
\begin{aligned}
V= & -\mu_{1}^{2}\left|\hat{\Sigma}_{L}\right|^{2}+\lambda_{1}\left|\hat{\Sigma}_{L}\right|^{4}-\mu_{2}^{2}\left|\hat{\Sigma}_{R}\right|^{2}+\lambda_{2}\left|\hat{\Sigma}_{R}\right|^{4}-\mu_{3}^{2}|\hat{\Sigma}|^{2}+\lambda_{3}\left(\hat{\Sigma} \hat{\Sigma}^{\dagger}\right)^{2}+\lambda_{3}^{\prime}\left|\hat{\Sigma} \hat{\Sigma}^{\dagger}\right|^{2}-\mu_{4}^{2}\left|\hat{H}_{d}\right|^{2}+\lambda_{4}\left(\hat{H}_{d} \hat{H}_{d}^{\dagger}\right)^{2}+\lambda_{4}^{\prime}\left|\hat{H}_{d} \hat{H}_{d}^{\dagger}\right|^{2} \\
& -\mu_{5}^{2}\left|\hat{H}_{u}\right|^{2}+\lambda_{5}\left(\hat{H}_{u} \hat{H}_{u}^{\dagger}\right)^{2}+\lambda_{5}^{\prime}\left|\hat{H}_{u} \hat{H}_{u}^{\dagger}\right|^{2}+\lambda_{12}\left|\hat{\Sigma}_{L}\right|^{2}\left|\hat{\Sigma}_{R}\right|^{2}+\lambda_{13}\left|\hat{\Sigma}_{L}\right|^{2}|\hat{\Sigma}|^{2}+\lambda_{14}\left|\hat{\Sigma}_{L}\right|^{2}\left|\hat{H}_{d}\right|^{2}+\lambda_{15}\left|\hat{\Sigma}_{L}\right|^{2}\left|\hat{H}_{u}\right|^{2}+\lambda_{23}\left|\hat{\Sigma}_{R}\right|^{2}|\hat{\Sigma}|^{2} \\
& +\lambda_{24}\left|\hat{\Sigma}_{R}\right|^{2}\left|\hat{H}_{d}\right|^{2}+\lambda_{25}\left|\hat{\Sigma}_{R}\right|^{2}\left|\hat{H}_{u}\right|^{2}+\lambda_{34}|\hat{\Sigma}|^{2}\left|\hat{H}_{d}\right|^{2}+\lambda_{35}|\hat{\Sigma}|^{2}\left|\hat{H}_{u}\right|^{2}+\lambda_{45}\left|\hat{H}_{d}\right|^{2}\left|\hat{H}_{u}\right|^{2}+\lambda_{13}^{\prime}\left|\hat{\Sigma}_{L} \hat{\Sigma}\right|^{2}+\lambda_{14}^{\prime}\left|\hat{\Sigma}_{L}^{\dagger} \hat{H}_{d}\right|^{2} \\
& +\lambda_{15}^{\prime}\left|\hat{\Sigma}_{L}^{\dagger} \hat{H}_{u}\right|^{2}+\lambda_{23}^{\prime}\left|\hat{\Sigma}_{R}^{\dagger} \hat{\Sigma}\right|^{2}+\lambda_{24}^{\prime}\left|\hat{\Sigma}_{R} \hat{H}_{d}\right|^{2}+\lambda_{25}^{\prime}\left|\hat{\Sigma}_{R} \hat{H}_{u}\right|^{2}+\lambda_{34}^{\prime}\left|\hat{\Sigma} \hat{H}_{d}\right|^{2}+\lambda_{35}^{\prime}\left|\hat{\Sigma} \hat{H}_{u}\right|^{2}+\lambda_{45}^{\prime}\left|\hat{H}_{d}^{\dagger} \hat{H}_{u}\right|^{2}+\lambda_{34}^{\prime \prime} \operatorname{Tr}\left(\hat{\Sigma} \hat{H}_{d} \hat{H}_{d}^{\dagger} \hat{\Sigma}^{\dagger}\right) \\
& +\lambda_{35}^{\prime \prime} \operatorname{Tr}\left(\hat{\Sigma} \hat{H}_{u} \hat{H}_{u}^{\dagger} \hat{\Sigma}^{\dagger}\right)+\lambda_{45}^{\prime \prime} \operatorname{Tr}\left(\hat{H}_{d}^{\dagger} \hat{H}_{u} \hat{H}_{u}^{\dagger} \hat{H}_{d}\right)+\left[\lambda_{345}^{\prime}\left(\hat{\Sigma} \hat{H}_{d}\right)\left(\hat{\Sigma} \hat{H}_{u}\right)+\lambda_{345}^{\prime \prime} \operatorname{Tr}\left(\hat{\Sigma} \hat{H}_{d} \hat{\Sigma} \hat{H}_{u}\right)\right. \\
& \left.+\lambda_{345}^{\prime \prime \prime} \operatorname{Tr}\left(\hat{H}_{d} \hat{\Sigma} \hat{\Sigma} \hat{H}_{u}\right)+\kappa \hat{\Sigma}_{L} \hat{\Sigma} \hat{\Sigma}_{R}^{\dagger}+\text { H.c. }\right],
\end{aligned}
$$

where we have adopted the notation $\left|\hat{\Sigma}_{L}\right|^{2} \equiv\left(\hat{\Sigma}_{L}\right)_{\alpha_{L}}\left(\hat{\Sigma}_{L}^{\dagger}\right)^{\alpha_{L}},|\hat{\Sigma}|^{2} \equiv(\hat{\Sigma})_{\alpha_{R}}^{\alpha_{L}}\left(\hat{\Sigma}^{\dagger}\right)_{\alpha_{L}}^{\alpha_{R}}, \quad\left(\hat{\Sigma} \hat{H}_{d}\right) \equiv(\hat{\Sigma})_{\alpha_{R}}^{\alpha_{L}}\left(\hat{H}_{d}\right)_{\alpha_{L}}^{\alpha_{R}},\left|\hat{\Sigma}_{L} \hat{\Sigma}\right|^{2} \equiv$ $\left(\hat{\Sigma}_{L}\right)_{\alpha_{L}}(\hat{\Sigma})_{\alpha_{R}}^{\alpha_{L}}\left(\hat{\Sigma}^{\dagger}\right)_{\beta_{L}}^{\alpha_{R}}\left(\hat{\Sigma}_{L}^{\dagger}\right)^{\beta_{L}},\left|\hat{\Sigma} \hat{H}_{d}\right|^{2} \equiv(\hat{\Sigma})_{\alpha_{R}}^{\alpha_{L}}\left(\hat{H}_{d}\right)_{\beta_{L}}^{\alpha_{R}}\left(\hat{H}_{d}^{\dagger}\right)_{\beta_{R}}^{\beta_{L}}\left(\hat{\Sigma}^{\dagger}\right)_{\alpha_{L}}^{\beta_{R}}, \operatorname{Tr}\left(\hat{\Sigma} \hat{H}_{d} \hat{\Sigma} \hat{H}_{u}\right) \equiv(\hat{\Sigma})_{\alpha_{R}}^{\alpha_{L}}\left(\hat{H}_{d}\right)_{\beta_{L}}^{\alpha_{R}}(\hat{\Sigma})_{\beta_{R}}^{\beta_{L}}\left(\hat{H}_{u}\right)_{\alpha_{L}}^{\beta_{R}}$, etc.

Let us consider $\left\langle\hat{\Sigma}_{L}\right\rangle,\left\langle\hat{\Sigma}_{R}\right\rangle$ and $\langle\hat{\Sigma}\rangle$. Via a suitable $\mathrm{SU}(4)_{L}$ and $\mathrm{SU}(4)_{R}$ transformation, it is possible to bring $\left\langle\hat{\Sigma}_{L}\right\rangle$ and $\left\langle\hat{\Sigma}_{R}\right\rangle$ to the form

$$
\left\langle\hat{\Sigma}_{L}\right\rangle=\frac{v_{L}}{\sqrt{2}}\left(\begin{array}{l}
0 \\
0 \\
0 \\
1
\end{array}\right), \quad\left\langle\hat{\Sigma}_{R}\right\rangle=\frac{v_{R}}{\sqrt{2}}\left(\begin{array}{l}
0 \\
0 \\
0 \\
1
\end{array}\right),
$$

where $v_{L}$ and $v_{R}$ are real and positive.

The remaining $\mathrm{SU}(3)$ invariance can be utilized to obtain

$$
\langle\hat{\Sigma}\rangle=\left(\begin{array}{cccc}
a_{1} & 0 & 0 & b_{1} \\
0 & a_{2} & 0 & b_{2} \\
0 & 0 & a_{3} & b_{3} \\
c_{1} & c_{2} & c_{3} & d
\end{array}\right) .
$$

To argue that $\langle\hat{\Sigma}\rangle$ can be brought to the diagonal form as in Eq. (4), it is sufficient to consider the potential terms $|\hat{\Sigma}|^{2}$, $\left(\hat{\Sigma} \hat{\Sigma}^{\dagger}\right)^{2},\left|\hat{\Sigma} \hat{\Sigma}^{\dagger}\right|^{2},\left|\hat{\Sigma}_{L} \hat{\Sigma}\right|^{2},\left|\hat{\Sigma}_{R}^{\dagger} \hat{\Sigma}\right|^{2}$ and $\hat{\Sigma}_{L} \hat{\Sigma} \hat{\Sigma}_{R}^{\dagger}$. Since

$$
\begin{aligned}
\lambda_{13}^{\prime}\left|\hat{\Sigma}_{L} \hat{\Sigma}\right|^{2}= & \frac{1}{2} \lambda_{13}^{\prime} v_{L}^{2}\left(c_{1}^{2}+c_{2}^{2}+c_{3}^{2}+d^{2}\right), \\
\lambda_{23}^{\prime}\left|\hat{\Sigma}_{R}^{\dagger} \hat{\Sigma}\right|^{2}= & \frac{1}{2} \lambda_{23}^{\prime} v_{R}^{2}\left(b_{1}^{2}+b_{2}^{2}+b_{3}^{2}+d^{2}\right), \\
-\mu_{3}^{2}|\hat{\Sigma}|^{2}+\lambda_{3}\left(\hat{\Sigma} \hat{\Sigma}^{\dagger}\right)^{2}= & \lambda_{3}\left(a_{1}^{2}+a_{2}^{2}+a_{3}^{2}+b_{1}^{2}+b_{2}^{2}+b_{3}^{2}\right. \\
& \left.+c_{1}^{2}+c_{2}^{2}+c_{3}^{2}+d^{2}-v_{\Sigma}^{2}\right)^{2}-\lambda_{3} v_{\Sigma}^{4},
\end{aligned}
$$

the potential is minimized for $\langle\hat{\Sigma}\rangle=\operatorname{diag}\left(a_{1}, a_{2}, a_{3}, d\right)$. In addition, the terms

$$
\begin{aligned}
& \lambda_{3}^{\prime}\left|\hat{\Sigma} \hat{\Sigma}^{\dagger}\right|^{2}=\lambda_{3}^{\prime}\left(a_{1}^{4}+a_{2}^{4}+a_{3}^{4}+d^{4}\right), \\
& \kappa \hat{\Sigma}_{L} \hat{\Sigma} \hat{\Sigma}_{R}^{\dagger}=\frac{1}{2} \kappa v_{L} v_{R} d
\end{aligned}
$$

imply that the minimum occurs at $a_{1}=a_{2}=a_{3}$. Finally, we are free to choose $\kappa$ to be real and negative, which through an appropriate redefinition of $\hat{\Sigma}$ leads to real $d>0$; therefore 


$$
\langle\hat{\Sigma}\rangle=\frac{v_{\Sigma}}{\sqrt{2}}\left(\begin{array}{cccc}
1 & 0 & 0 & 0 \\
0 & 1 & 0 & 0 \\
0 & 0 & 1 & 0 \\
0 & 0 & 0 & z
\end{array}\right)
$$

with $z$ being real and positive. Note that only one of the parameters $\lambda_{345}^{\prime}, \lambda_{345}^{\prime \prime}$ and $\lambda_{345}^{\prime \prime \prime}$ can be made real by a field redefinition. If any of the other two has a nonzero imaginary part, the scalar potential is $C P$-violating. A rigorous minimization procedure is beyond the scope of this work.

\section{APPENDIX B: SCALAR MASSES}

To show that Eq. (9) can be satisfied, it is again sufficient to consider only a few terms in the scalar potential. In terms of hard masses, the relevant part of the Lagrangian is

$$
\begin{gathered}
\mathcal{L}_{m} \supset M_{d}^{2}\left|\hat{H}_{d}\right|^{2}+M_{u}^{2}\left|\hat{H}_{u}\right|^{2}-\lambda_{24}^{\prime}\left|\hat{\Sigma}_{R} \hat{H}_{d}\right|^{2}-\lambda_{25}^{\prime}\left|\hat{\Sigma}_{R} \hat{H}_{u}\right|^{2} \\
-\lambda_{34}^{\prime}\left|\hat{\Sigma} \hat{H}_{d}\right|^{2}-\lambda_{35}^{\prime}\left|\hat{\Sigma} \hat{H}_{u}\right|^{2}-\lambda_{345}^{\prime}\left(\hat{\Sigma} \hat{H}_{d}\right)\left(\hat{\Sigma} \hat{H}_{u}\right) .
\end{gathered}
$$

This results in the masses for the color octets and triplets,

$$
\begin{aligned}
& m_{O_{1}}=m_{T_{1}}=m_{T_{2}} \equiv M_{d}^{2}, \\
& m_{O_{2}}=m_{T_{3}}=m_{T_{4}} \equiv M_{u}^{2} .
\end{aligned}
$$

The mass squared matrix for the fields $S_{1,2,3,4}$ is

$$
\mathcal{M}_{S}^{2}=\left(\begin{array}{cccc}
M_{d}^{2}+a_{d} & 0 & \frac{1}{2} \lambda_{345}^{\prime} z^{2} v_{\Sigma}^{2} & \frac{\sqrt{3}}{2} \lambda_{345}^{\prime} z v_{\Sigma}^{2} \\
0 & M_{d}^{2}+b_{d} & \frac{\sqrt{3}}{2} \lambda_{345}^{\prime} z v_{\Sigma}^{2} & \frac{3}{2} \lambda_{345}^{\prime} v_{\Sigma}^{2} \\
\frac{1}{2} \lambda_{345}^{\prime} z^{2} v_{\Sigma}^{2} & \frac{\sqrt{3}}{2} \lambda_{345}^{\prime} z v_{\Sigma}^{2} & M_{u}^{2}+a_{u} & 0 \\
\frac{\sqrt{3}}{2} \lambda_{345}^{\prime} z v_{\Sigma}^{2} & \frac{3}{2} \lambda_{345}^{\prime} v_{\Sigma}^{2} & 0 & M_{u}^{2}+b_{u}
\end{array}\right),
$$

where

$$
\begin{aligned}
a_{d} & =\frac{1}{2} \lambda_{24}^{\prime} v_{R}^{2}+\frac{1}{2} \lambda_{34}^{\prime} z^{2} v_{\Sigma}^{2}, & b_{d} & =\frac{3}{2} \lambda_{34}^{\prime} v_{\Sigma}^{2}, \\
a_{u} & =\frac{1}{2} \lambda_{25}^{\prime} v_{R}^{2}+\frac{1}{2} \lambda_{35}^{\prime} z^{2} v_{\Sigma}^{2}, & b_{u} & =\frac{3}{2} \lambda_{35}^{\prime} v_{\Sigma}^{2} .
\end{aligned}
$$

We have verified that there exists a class of solutions with only one linear combination of the four scalars being light. To reproduce the SM fermion masses while keeping the Yukawas perturbative, it is sufficient to have the light mass eigenstate, identified with the SM Higgs, given by

$$
H=-c_{e} S_{1}-c_{d} S_{2}+c_{\nu} S_{3}+c_{u} S_{4},
$$

where $c_{u} \approx 1 \gg c_{d} \gg c_{\nu}$ and $1 \gg c_{e} \gg c_{\nu}$, with the ratio $c_{d}: c_{e} \approx m_{b}: m_{\tau}$.

\section{APPENDIX C: FLAVOR CONSTRAINTS: MODEL-INDEPENDENT ANALYSIS}

The general form of the Lagrangian describing interactions of vector leptoquarks $(3,1)_{2 / 3}$ with fermions is given by

$$
\begin{gathered}
\mathcal{L} \supset \sum_{\alpha} X_{\mu}^{(\alpha)}\left[f_{i j(\alpha)}^{L u}\left(\bar{u}^{i} \gamma^{\mu} P_{L} \nu^{j}\right)+f_{i j(\alpha)}^{R u}\left(\bar{u}^{i} \gamma^{\mu} P_{R} \nu^{j}\right)\right. \\
\left.+f_{i j(\alpha)}^{L d}\left(\bar{d}^{i} \gamma^{\mu} P_{L} e^{j}\right)+f_{i j(\alpha)}^{R d}\left(\bar{d}^{i} \gamma^{\mu} P_{R} e^{j}\right)\right]
\end{gathered}
$$

where the field $X_{\mu}^{(\alpha)}$ corresponds to a leptoquark with mass $M_{\alpha}$. The resulting contributions to rare processes are listed below, along with the most severe experimental bounds.

The numerical values for particle masses and lifetimes were adopted from PDG [71]. The single-particle state normalization chosen is

$$
\left\langle\vec{p} \mid \vec{p}^{\prime}\right\rangle=2 E(2 \pi)^{3} \delta^{(3)}\left(\vec{p}-\vec{p}^{\prime}\right)
$$

and the decay constant $f_{\mathcal{M}}$ for a meson consisting of quarks/antiquarks $q_{1}, q_{2}$ is defined via

$$
\begin{aligned}
\left\langle 0\left|\bar{q}_{1} \gamma^{5} q_{2}\right| \mathcal{M}(p)\right\rangle & =-i f_{\mathcal{M}} \frac{m_{\mathcal{M}}^{2}}{m_{q_{1}}+m_{q_{2}}}, \\
\left\langle 0\left|\bar{q}_{1} \gamma^{\mu} \gamma^{5} q_{2}\right| \mathcal{M}(p)\right\rangle & =i f_{\mathcal{M}} p^{\mu} .
\end{aligned}
$$

The following values for the meson decay constants were adopted from the PDG,

$$
\begin{aligned}
& f_{\pi^{+}}=130 \mathrm{MeV}, \quad f_{K_{L}^{0}}=f_{K^{+}}=156 \mathrm{MeV}, \\
& f_{B^{0}}=191 \mathrm{MeV}, \quad f_{B^{+}}=187 \mathrm{MeV}, \quad f_{B_{s}^{0}}=227 \mathrm{MeV},
\end{aligned}
$$

and were obtained by averaging the lattice results.

The other decay constants needed in our analysis are

$$
\begin{array}{rlrl}
f_{\phi} & \approx 238 \mathrm{MeV}, & & f_{\Upsilon(1 S)} \approx 700 \mathrm{MeV}, \\
f_{\Upsilon(2 S)} \approx 496 \mathrm{MeV}, & & f_{\Upsilon(3 S)} \approx 430 \mathrm{MeV},
\end{array}
$$

where $f_{\phi}$ was determined from the lattice [72] and $f_{\Upsilon(n S)}$ $(n=1,2,3)$ were extracted from the experimental results for $\Upsilon(n S) \rightarrow \ell^{-} \ell^{+}$(see [69,73]).

Below, we present constraints on a general model with $(3,1)_{2 / 3}$ vector leptoquarks described by the Lagrangian (C1). The constraints arise from the following processes:

1. Neutral meson decays to two charged leptons
a. Neutral kaon decays
b. Neutral $B$ meson decays
c. $\Upsilon$ decays

2. Charged meson decays to a charged lepton and neutrino
a. Charged pion decays
b. Charged kaon decays 
3. Charged meson three-body decays to a meson and charged leptons

a. Charged kaon decays

b. Charged $B$ meson decays

4. Tau decays

5. Radiative charged lepton decay

6. $l_{i}^{+} \rightarrow l_{j}^{+}$conversion

The relevant formulas are listed below. The numbering of the sections matches that in the table of contents above.

\section{Neutral meson decays to two charged leptons}

The leptoquark contribution to the decay of a pseudoscalar meson $\mathcal{M}$ with mass $m_{\mathcal{M}}$ to two charged leptons, $l_{i}^{+}$ with mass $m_{i}$ and $l_{j}^{-}$with mass $m_{j}$, is given by

$$
\begin{aligned}
\Gamma\left(\mathcal{M} \rightarrow l_{i}^{+} l_{j}^{-}\right)_{X}= & \frac{m_{\mathcal{M}} f_{\mathcal{M}}^{2}}{64 \pi}\left[A_{i j}\left(1-\frac{m_{i}^{2}+m_{j}^{2}}{m_{\mathcal{M}}^{2}}\right)+B_{i j} \frac{4 m_{i} m_{j}}{m_{\mathcal{M}}^{2}}\right] \\
& \times \sqrt{\left[1-\frac{\left(m_{i}+m_{j}\right)^{2}}{m_{\mathcal{M}}^{2}}\right]\left[1-\frac{\left(m_{i}-m_{j}\right)^{2}}{m_{\mathcal{M}}^{2}}\right]},
\end{aligned}
$$

where

$$
\begin{aligned}
A_{i j} \equiv & \left|\sum_{\alpha} \frac{a_{i j(\alpha)}^{L R}}{M_{\alpha}^{2}}\right|^{2}+\left|\sum_{\alpha} \frac{a_{i j(\alpha)}^{R L}}{M_{\alpha}^{2}}\right|^{2}, \\
B_{i j} \equiv & \sum_{\alpha, \beta} \frac{\operatorname{Re}\left[a_{i j(\alpha)}^{L R} a_{i j(\beta)}^{R L *}\right]}{M_{\alpha}^{2} M_{\beta}^{2}}, \\
a_{i j(\alpha)}^{L R\left(K_{L}^{0}\right)} \equiv & \frac{1}{\sqrt{2}}\left[m_{i} f_{1 i(\alpha)}^{R d} f_{2 j(\alpha)}^{R d *}+m_{j} f_{1 i(\alpha)}^{L d} f_{2 j(\alpha)}^{L d *}\right. \\
& \left.+\frac{2 m_{K^{0}}^{2} Q}{m_{s}+m_{d}} f_{1 i(\alpha)}^{L d} f_{2 j(\alpha)}^{R d *}\right]+(1 \leftrightarrow 2), \\
a_{i j(\alpha)}^{L R\left(B^{0}\right)} \equiv & m_{i} f_{1 i(\alpha)}^{R d *} f_{3 j(\alpha)}^{R d}+m_{j} f_{1 i(\alpha)}^{L d *} f_{3 j(\alpha)}^{L d} \\
& +\frac{2 m_{B^{0}}^{2} Q}{m_{b}+m_{d}} f_{1 i(\alpha)}^{L d *} f_{3 j(\alpha)}^{R d}, \\
a_{i j(\alpha)}^{L R\left(B_{s}^{0}\right)} \equiv & m_{i} f_{2 i(\alpha)}^{R d *} f_{3 j(\alpha)}^{R d}+m_{j} f_{2 i(\alpha)}^{L d *} f_{3 j(\alpha)}^{L d} \\
& +\frac{2 m_{B_{s}^{0}}^{2} Q}{m_{b}+m_{s}} f_{2 i(\alpha)}^{L d *} f_{3 j(\alpha)}^{R d}, \\
a_{i j(\alpha)}^{R L} \equiv & a_{i j(\alpha)}^{L R}(L \leftrightarrow R) .
\end{aligned}
$$

In Eq. (C7) the quark masses $m_{q}$ and the factor $Q$ depend on the energy scale, $m_{q}=m_{q}(\mu)$ and $Q=Q(\mu)$, with $Q(\mu)$ given by the formula

$$
Q(\mu)=\left[\frac{\alpha^{(6)}\left(m_{t}\right)}{\alpha^{(6)}\left(M_{X_{L}}\right)}\right]^{\frac{4}{7}}\left[\frac{\alpha^{(5)}\left(m_{b}\right)}{\alpha^{(5)}\left(m_{t}\right)}\right]^{\frac{12}{23}}\left[\frac{\alpha^{(4)}(\mu)}{\alpha^{(4)}\left(m_{b}\right)}\right]^{\frac{12}{25}},
$$

applicable for $m_{b}>\mu>m_{c}$. The coupling constant $\alpha$ is calculated from

$$
\alpha^{\left(N_{f}\right)}(\mu, \Lambda)=\frac{4 \pi}{\left(11-2 N_{f} / 3\right) \log \left(\mu^{2} / \Lambda^{2}\right)},
$$

where $N_{f}$ is the number of quark flavors at a given scale, by matching

$$
\begin{gathered}
\alpha^{(6)}\left(m_{t}\right) \equiv \alpha^{(6)}\left(m_{t}, \Lambda_{6}\right)=\alpha^{(5)}\left(m_{t}, \Lambda_{5}\right) \equiv \alpha^{(5)}\left(m_{t}\right), \\
\alpha^{(5)}\left(m_{b}\right) \equiv \alpha^{(5)}\left(m_{b}, \Lambda_{5}\right)=\alpha^{(4)}\left(m_{b}, \Lambda_{4}\right) \equiv \alpha^{(4)}\left(m_{b}\right) .
\end{gathered}
$$

The ratio $Q(\mu) / m_{q}(\mu)$ is a renormalization group invariant. Adopting the PDG values for the quark masses at $\mu=$ $2 \mathrm{GeV}$ and for the strong coupling constant at $\mu=M_{Z}$ [71], the value of $Q$ depends only on the leptoquark mass scale through

$$
Q(2 \mathrm{GeV})=\frac{0.45}{\left[\alpha^{(6)}\left(M_{X_{L}}\right)\right]^{4 / 7}} .
$$

As evident from Eq. (C7), the constraints on the leptoquark contribution to the branching fraction of kaon and $B$ meson decays are much weaker when the leptoquarks have only LH or only RH interactions with SM fermions, as opposed to models with both $\mathrm{LH}$ and $\mathrm{RH}$ interactions. The bounds on the branching fraction are milder by a factor of

$$
\sqrt{2 m_{\mathcal{M}}^{2} Q /\left(m_{l} m_{q}\right)}
$$

which is reflected by the much weaker constraints on the leptoquark mass in our model compared to generic leptoquark models (see Appendix D).

For the majority of decays considered here only the upper bound on the rate was experimentally established. However, in the four cases $K_{L}^{0} \rightarrow e^{+} e^{-}, K_{L}^{0} \rightarrow \mu^{+} \mu^{-}, B^{0} \rightarrow$ $\mu^{+} \mu^{-}$and $B_{s}^{0} \rightarrow \mu^{+} \mu^{-}$nonzero rates have been measured. For those particular decays not only the pure leptoquark contribution is relevant, but also the interference effects with the SM short-distance (SD) contribution. This can be taken into account by making the following substitution in the expressions for $A_{i j}$ and $B_{i j}$ in Eq. (C7):

$$
\sum_{\alpha} \frac{a_{i j(\alpha)}^{L R}}{M_{\alpha}^{2}} \rightarrow \sqrt{\frac{64 \pi \Gamma\left(\mathcal{M} \rightarrow l_{i}^{+} l_{j}^{-}\right)_{\mathrm{SM}}^{\mathrm{SD}}}{m_{\mathcal{M}} f_{\mathcal{M}}^{2}}} \delta_{i j} \pm \sum_{\alpha} \frac{a_{i j(\alpha)}^{L R}}{M_{\alpha}^{2}},
$$

where the $+/-$ depends on the decay considered and corresponds to the SM short-distance amplitude for $\mathcal{M} \rightarrow$ $l_{i}^{+} l_{j}^{-}$being negative/positive. The leptoquark-induced contribution is then obtained by subtracting off the pure SM part. 


\section{a. Neutral kaon decays}

The decays $K_{L}^{0} \rightarrow e^{ \pm} \mu^{\mp}$ are absent in the SM and the constraint on the leptoquark mass is derived directly from the experimental bound on the branching fraction, $\mathrm{Br}_{X} \lesssim \Delta \mathrm{Br}$. The rates for $K_{L}^{0} \rightarrow e^{+} e^{-}, \mu^{+} \mu^{-}$were measured $[41,71]$. They are dominated by long-distance SM effects [74,75]. For $K_{L}^{0} \rightarrow e^{+} e^{-}$the experimental branching fraction $\left(8.7_{-4.1}^{+5.7}\right) \times 10^{-12}$ [41] agrees well with the SM long-distance estimate of $(9.0 \pm 0.5) \times 10^{-12}$ [74]. In that case we use the experimental uncertainty for the measured branching fraction as the upper bound for the leptoquark contribution. For $K_{L}^{0} \rightarrow \mu^{+} \mu^{-}$the measured branching fraction is $(6.84 \pm 0.11) \times 10^{-9}$ [43], but it was shown that the short-distance SM contribution is only $0.9 \times 10^{-9}$ [74], whereas the upper bound on the total short-distance contribution is $2.5 \times 10^{-9}$ [75].

The constraints below reflect the most conservative bound on the leptoquark mass obtained using Eq. (C6) (the branching fractions were left in explicitly for easier use of the formulas given future experimental improvements):

$$
\begin{aligned}
& \frac{A_{11}^{\left(K_{L}^{0}\right)}}{m_{K^{0}}^{2}} \lesssim\left[\frac{\operatorname{Br}\left(K_{L}^{0} \rightarrow e^{+} e^{-}\right)}{5.7 \times 10^{-12}}\right](672 \mathrm{TeV})^{-4}, \quad[41] \\
& \frac{A_{12}^{\left(K_{L}^{0}\right)}+A_{21}^{\left(K_{L}^{0}\right)}}{m_{K^{0}}^{2}} \lesssim\left[\frac{\operatorname{Br}\left(K_{L}^{0} \rightarrow e^{ \pm} \mu^{\mp}\right)}{4.7 \times 10^{-12}}\right](689 \mathrm{TeV})^{-4}, \quad[42]
\end{aligned}
$$

$$
\begin{aligned}
\frac{{A^{\prime}}_{22}^{\left(K_{L}^{0}\right)}+0.2{B^{\prime}}_{22}^{\left(K_{L}^{0}\right)}}{m_{K^{0}}^{2}} & \lesssim\left[\frac{\operatorname{Br}\left(K_{L}^{0} \rightarrow \mu^{+} \mu^{-}\right)}{2.5 \times 10^{-9}}\right] \\
& \times(140 \mathrm{TeV})^{-4},[75]
\end{aligned}
$$

where $A_{22}^{\prime\left(K_{L}^{0}\right)}$ is given by $A_{22}^{\left(K_{L}^{0}\right)}$ with the substitution (C12) with $\Gamma\left(K_{L}^{0} \rightarrow \mu^{+} \mu^{-}\right)_{\mathrm{SM}}^{\mathrm{SD}}=0.9 \times 10^{-9}$, and similarly for $B_{22}^{\prime\left(K_{L}^{0}\right)}$.

\section{b. Neutral B meson decays}

For most of the $B^{0}$ and $B_{s}^{0}$ decays only the limit on the branching fraction is determined; therefore the bounds on leptoquark parameters are derived using $\mathrm{Br}_{X} \lesssim \Delta \mathrm{Br}$. In the case of $B^{0} \rightarrow \mu^{+} \mu^{-}$and $B_{s}^{0} \rightarrow \mu^{+} \mu^{-}$the branching fractions were actually measured, $\operatorname{Br}\left(B^{0} \rightarrow \mu^{+} \mu^{-}\right)=\left(1.6_{-1.4}^{+1.6}\right) \times 10^{-10}$ [71] and $\operatorname{Br}\left(B_{s}^{0} \rightarrow \mu^{+} \mu^{-}\right)=\left(3.0 \pm 0.6_{-0.2}^{+0.3}\right) \times 10^{-9}$ [53], and they are dominated by short-distance SM effects. We arrive at the following set of constraints:

$$
\begin{aligned}
& \frac{A_{11}^{\left(B^{0}\right)}}{m_{B^{0}}^{2}} \lesssim\left[\frac{\operatorname{Br}\left(B^{0} \rightarrow e^{+} e^{-}\right)}{8.3 \times 10^{-8}}\right](29.4 \mathrm{TeV})^{-4}, \\
& \frac{A_{12}^{\left(B^{0}\right)}+A_{21}^{\left(B^{0}\right)}}{m_{B^{0}}^{2}} \lesssim\left[\frac{\operatorname{Br}\left(B^{0} \rightarrow e^{ \pm} \mu^{\mp}\right)}{1.0 \times 10^{-9}}\right] \\
& \times(88.6 \mathrm{TeV})^{-4} \\
& \frac{A_{22}^{\prime\left(B^{0}\right)}}{m_{B^{0}}^{2}} \lesssim\left[\frac{\operatorname{Br}\left(B^{0} \rightarrow \mu^{+} \mu^{-}\right)}{1.6 \times 10^{-10}}\right](140 \mathrm{TeV})^{-4}, \\
& \frac{A_{13}^{\left(B^{0}\right)}+A_{31}^{\left(B^{0}\right)}}{m_{B^{0}}^{2}} \lesssim\left[\frac{\operatorname{Br}\left(B^{0} \rightarrow e^{ \pm} \tau^{\mp}\right)}{2.8 \times 10^{-5}}\right] \\
& \times(6.4 \mathrm{TeV})^{-4}, \quad[50]
\end{aligned}
$$

$$
\begin{aligned}
\frac{A_{23}^{\left(B^{0}\right)}+A_{32}^{\left(B^{0}\right)}}{m_{B^{0}}^{2}} \lesssim & {\left[\frac{\operatorname{Br}\left(B^{0} \rightarrow \mu^{ \pm} \tau^{\mp}\right)}{2.2 \times 10^{-5}}\right] } \\
& \times(6.8 \mathrm{TeV})^{-4}, \quad[50] \\
\frac{A_{33}^{\left(B^{0}\right)}+0.59 B_{33}^{\left(B^{0}\right)}}{m_{B^{0}}^{2}} \lesssim & {\left[\frac{\operatorname{Br}\left(B^{0} \rightarrow \tau^{+} \tau^{-}\right)}{2.1 \times 10^{-3}}\right] } \\
& \times(2.0 \mathrm{TeV})^{-4}, \quad[54]
\end{aligned}
$$

$$
\frac{A_{11}^{\left(B_{s}^{0}\right)}}{m_{B_{s}^{0}}^{2}} \lesssim\left[\frac{\operatorname{Br}\left(B_{s}^{0} \rightarrow e^{+} e^{-}\right)}{2.8 \times 10^{-7}}\right](23.9 \mathrm{TeV})^{-4},
$$

$$
\begin{aligned}
\frac{A_{12}^{\left(B_{s}^{0}\right)}+A_{21}^{\left(B_{s}^{0}\right)}}{m_{B_{s}^{0}}^{2}} \lesssim & {\left[\frac{\operatorname{Br}\left(B_{s}^{0} \rightarrow e^{ \pm} \mu^{\mp}\right)}{5.4 \times 10^{-9}}\right] } \\
& \times(64.1 \mathrm{TeV})^{-4}, \quad[52]
\end{aligned}
$$

$$
\begin{aligned}
& \frac{A_{22}^{\prime\left(B_{s}^{0}\right)}}{m_{B_{s}^{0}}^{2}} \lesssim\left[\frac{\operatorname{Br}\left(B_{s}^{0} \rightarrow \mu^{+} \mu^{-}\right)}{0.7 \times 10^{-9}}\right](107 \mathrm{TeV})^{-4}, \\
& \frac{A_{33}^{\left(B_{s}^{0}\right)}+0.56 B_{33}^{\left(B_{s}^{0}\right)}}{m_{B_{s}^{0}}^{2}} \lesssim {\left[\frac{\operatorname{Br}\left(B_{s}^{0} \rightarrow \tau^{+} \tau^{-}\right)}{6.8 \times 10^{-3}}\right] } \\
& \times(1.7 \mathrm{TeV})^{-4},
\end{aligned}
$$


where $A_{22}^{\prime\left(B^{0}\right)}$ is given by $A_{22}^{\left(B^{0}\right)}$ with the substitution (C12) with $\Gamma\left(B^{0} \rightarrow \mu^{+} \mu^{-}\right)_{\mathrm{SM}}^{\mathrm{SD}}=1.6 \times 10^{-10}$, and similarly for $A_{22}^{\prime\left(B_{s}^{0}\right)}$ with $\Gamma\left(B_{s}^{0} \rightarrow \mu^{+} \mu^{-}\right)_{\mathrm{SM}}^{\mathrm{SD}}=3.0 \times 10^{-9}$. We listed the constraint on the leptoquark contribution to $\operatorname{Br}\left(B_{s}^{0} \rightarrow\right.$ $\mu^{+} \mu^{-}$) for completeness, but this branching fraction is actually determined by the fit that yields $\Delta C_{9}$ and $\Delta C_{10}$ in Eq. (27).

\section{c. $\Upsilon$ decays}

This set of constraints arises from the vector meson decays $\Upsilon(n S) \rightarrow e^{ \pm} \tau^{\mp}$ and $\Upsilon(n S) \rightarrow \mu^{ \pm} \tau^{\mp}$. Neglecting the electron and muon mass, the corresponding branching fraction is given by Eq. (C6) (only the term with $A_{i j}$ is nonzero), where

$$
\begin{aligned}
a_{i j(\alpha)}^{L R(\Upsilon(n S))} \equiv & \sqrt{\frac{8}{3}\left(1+\frac{m_{\tau}^{2}}{2 m_{\Upsilon(n S)}^{2}}\right)}\left(m_{i} f_{3 i(\alpha)}^{R d *} f_{3 j(\alpha)}^{R d}\right. \\
& \left.+m_{j} f_{3 i(\alpha)}^{L d *} f_{3 j(\alpha)}^{L d}+\frac{m_{\Upsilon(n S)}^{2} Q}{m_{b}} f_{3 i(\alpha)}^{L d *} f_{3 j(\alpha)}^{R d}\right), \\
a_{i j(\alpha)}^{R L(\Upsilon(n S))} \equiv & a_{i j(\alpha)}^{L R(\Upsilon(n S))}(L \leftrightarrow R) .
\end{aligned}
$$

Of all $\Upsilon(n S) \rightarrow \ell^{ \pm} \tau^{\mp}$ decays, the $\Upsilon(3 S) \rightarrow \mu^{ \pm} \tau^{\mp}$ gives the tightest constraints. From the corresponding experimental bounds we have

$$
\begin{aligned}
\frac{A_{23}^{(\Upsilon(3 S))}+A_{32}^{(\Upsilon(3 S))}}{m_{\Upsilon(3 S)}^{2}} \lesssim & {\left[\frac{\operatorname{Br}\left(\Upsilon(3 S) \rightarrow \mu^{ \pm} \tau^{\mp}\right)}{3.1 \times 10^{-6}}\right] } \\
& \times(0.5 \mathrm{TeV})^{-4}, \quad[52]
\end{aligned}
$$

which, however, is still much weaker than all other constraints considered here.

\section{Charged meson decays to a charged lepton and a neutrino}

Decays of mesons to a charged lepton and a neutrino exist in the SM. The leading order leptoquark contribution comes from interference effects. The theoretical uncertainty in the SM calculation is reduced by taking ratios of decay rates,

$$
\frac{\Gamma\left(\mathcal{M} \rightarrow l_{i}^{+} \nu\right)}{\Gamma\left(\mathcal{M} \rightarrow l_{i^{\prime}}^{+} \nu\right)}=\left.\frac{\Gamma\left(\mathcal{M} \rightarrow l_{i}^{+} \nu\right)}{\Gamma\left(\mathcal{M} \rightarrow l_{i^{\prime}}^{+} \nu\right)}\right|_{\mathrm{SM}}\left(1+\frac{D_{i^{\prime}}-D_{i}}{\sqrt{2} G_{F}}\right)
$$

where

$$
D_{i} \equiv \sum_{\alpha ; j} \frac{1}{M_{\alpha}^{2}} \operatorname{Re}\left[d_{i j(\alpha)}^{L R}+d_{i j(\alpha)}^{R L}\right]
$$

For the case of Dirac neutrinos,

$$
\begin{aligned}
d_{i j(\alpha)}^{L R\left(\pi^{+}\right)} & \equiv \frac{U_{i j}}{V_{u d}}\left[f_{1 i(\alpha)}^{R d} f_{1 j(\alpha)}^{R u *}+\frac{2 m_{\pi^{+}}^{2} Q}{m_{i}\left(m_{d}+m_{u}\right)} f_{1 i(\alpha)}^{L d} f_{1 j(\alpha)}^{R u *}\right], \\
d_{i j(\alpha)}^{L R\left(K^{+}\right)} & \equiv \frac{U_{i j}}{V_{u s}}\left[f_{1 i(\alpha)}^{R d} f_{2 j(\alpha)}^{R u *}+\frac{2 m_{K^{+}}^{2} Q}{m_{i}\left(m_{s}+m_{u}\right)} f_{1 i(\alpha)}^{L d} f_{2 j(\alpha)}^{R u *}\right], \\
d_{i j(\alpha)}^{R L} & \equiv d_{i j(\alpha)}^{L R}(L \leftrightarrow R),
\end{aligned}
$$

whereas for Majorana neutrinos the only nonzero terms are

$$
d_{i j(\alpha)}^{R L\left(\pi^{+}\right)} \equiv \frac{U_{i j}}{V_{u d}} f_{1 i(\alpha)}^{L d} f_{1 j(\alpha)}^{L u *}, \quad d_{i j(\alpha)}^{R L\left(K^{+}\right)} \equiv \frac{U_{i j}}{V_{s d}} f_{1 i(\alpha)}^{L d} f_{2 j(\alpha)}^{L u *} .
$$

The tightest bounds of this type originate from measurements of the branching fraction ratios:

$$
R\left(\pi^{+}\right) \equiv \frac{\Gamma\left(\pi^{+} \rightarrow e^{+} \nu\right)}{\Gamma\left(\pi^{+} \rightarrow \mu^{+} \nu\right)}, \quad R\left(K^{+}\right) \equiv \frac{\Gamma\left(K^{+} \rightarrow e^{+} \nu\right)}{\Gamma\left(K^{+} \rightarrow \mu^{+} \nu\right)} .
$$

\section{a. Charged pion decays}

The experimental measurement and the SM prediction yield

$$
\begin{aligned}
R\left(\pi^{+}\right) & =(1.2327 \pm 0.0023) \times 10^{-4} \quad[71], \\
R\left(\pi^{+}\right)_{\mathrm{SM}} & =(1.2352 \pm 0.0001) \times 10^{-4} \quad[76],
\end{aligned}
$$

which, given Eq. (C28), leads to

$$
\left|D_{1}^{\left(\pi^{+}\right)}-D_{2}^{\left(\pi^{+}\right)}\right| \lesssim(3.9 \mathrm{TeV})^{-2}
$$

\section{b. Charged kaon decays}

In this case,

$$
\begin{aligned}
& R\left(K^{+}\right)=(2.493 \pm 0.031) \times 10^{-5} \quad[46], \\
& R\left(K^{+}\right)_{\mathrm{SM}}=(2.477 \pm 0.001) \times 10^{-5} \quad[76],
\end{aligned}
$$

which results in

$$
\left|D_{1}^{\left(K^{+}\right)}-D_{2}^{\left(K^{+}\right)}\right| \lesssim(3.1 \mathrm{TeV})^{-2}
$$

\section{Charged meson three-body decays to a meson and charged leptons}

When the leptoquark has both $\mathrm{LH}$ and $\mathrm{RH}$ interactions with SM fermions, the three-body meson decays are less restrictive than the two-body decays. However, in the case of our model, with predominantly LH interactions, the bounds arising from $B^{+} \rightarrow K^{+} e^{ \pm} \mu^{\mp}$ impose the most severe constraints on the leptoquark mass. The corresponding decay rate is expressed in terms of the form factors $f_{+}\left(q^{2}\right)$ and $f_{0}\left(q^{2}\right)$ defined via 


$$
\begin{aligned}
\left\langle\mathcal{M}^{\prime}\left(p^{\prime}\right)\left|\bar{q}_{1} \gamma^{\mu} q_{2}\right| \overline{\mathcal{M}}(p)\right\rangle & =f_{+}\left(q^{2}\right)\left[p^{\mu}+p^{\prime \mu}-\frac{\Delta M^{2}}{q^{2}} q^{\mu}\right]+f_{0}\left(q^{2}\right) \frac{\Delta M^{2}}{q^{2}} q^{\mu}, \\
\left\langle\mathcal{M}^{\prime}\left(p^{\prime}\right)\left|\bar{q}_{1} q_{2}\right| \overline{\mathcal{M}}(p)\right\rangle & =f_{0}\left(q^{2}\right) \frac{\Delta M^{2}}{m_{q_{1}}-m_{q_{2}}}
\end{aligned}
$$

where the four-momentum transfer $q=p^{\prime}-p$ and the meson squared mass difference $\Delta M^{2}=m_{\mathcal{M}}^{2}-m_{\mathcal{M}^{\prime}}^{2}$. The contribution to the decay rate mediated by leptoquarks is

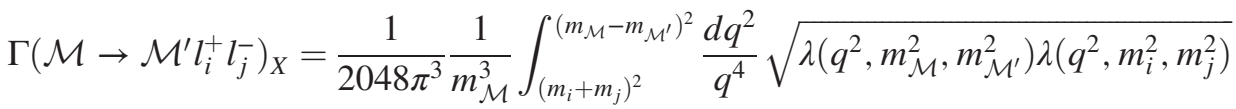

$$
\begin{aligned}
& \times\left\{\left[\frac{1}{3} N_{i j}^{+}\left(2 q^{2}-m_{i}^{2}-m_{j}^{2}-\frac{\left(m_{i}^{2}-m_{j}^{2}\right)^{2}}{q^{2}}\right)+2 N_{i j}^{-} m_{i} m_{j}\right] \lambda\left(q^{2}, m_{\mathcal{M}^{2}}^{2}, m_{\mathcal{M}^{\prime}}^{2}\right)\left|f_{+}\left(q^{2}\right)\right|^{2}\right. \\
& +\left[N_{i j}^{+}\left(m_{i}^{2}+m_{j}^{2}-\frac{\left(m_{i}^{2}-m_{j}^{2}\right)^{2}}{q^{2}}\right)-2 N_{i j}^{-} m_{i} m_{j}+4 P_{i j}^{+} q^{2}\left(q^{2}-m_{i}^{2}-m_{j}^{2}\right)-8 P_{i j}^{-} q^{2} m_{i} m_{j}\right. \\
& \left.\left.-4\left(R_{i j}^{+}+R_{i j}^{-}\right) m_{i}\left(q^{2}-m_{i}^{2}+m_{j}^{2}\right)+4\left(R_{i j}^{+}-R_{i j}^{-}\right) m_{j}\left(q^{2}+m_{i}^{2}-m_{j}^{2}\right)\right]\left(m_{\mathcal{M}}^{2}-m_{\mathcal{M}^{\prime}}^{2}\right)^{2}\left|f_{0}\left(q^{2}\right)\right|^{2}\right\},
\end{aligned}
$$

where

$$
\begin{aligned}
& \lambda(x, y, z) \equiv(x-y-z)^{2}-4 y z, \quad N_{i j}^{ \pm} \equiv\left|\sum_{\alpha} \frac{n_{i j(\alpha)}^{L L}+n_{i j(\alpha)}^{R R}}{M_{\alpha}^{2}}\right|^{2} \pm\left|\sum_{\alpha} \frac{n_{i j(\alpha)}^{L L}-n_{i j(\alpha)}^{R R}}{M_{\alpha}^{2}}\right|^{2}, \\
& P_{i j}^{ \pm} \equiv\left|\sum_{\alpha} \frac{p_{i j(\alpha)}^{L R}+p_{i j(\alpha)}^{R L}}{M_{\alpha}^{2}}\right|^{2} \pm\left|\sum_{\alpha} \frac{p_{i j(\alpha)}^{L R}-p_{i j(\alpha)}^{R L}}{M_{\alpha}^{2}}\right|^{2}, \quad R_{i j}^{ \pm} \equiv \sum_{\alpha, \beta} \frac{\operatorname{Re}\left[\left(n_{i j(\alpha)}^{L L} \pm n_{i j(\alpha)}^{R R}\right)\left(p_{i j(\beta)}^{L R} \pm p_{i j(\beta)}^{R L}\right)\right]}{M_{\alpha}^{2} M_{\beta}^{2}}, \\
& n_{i j(\alpha)}^{L L\left(K^{+}, \pi^{+}\right)}=f_{1 i(\alpha)}^{L d} f_{2 j(\alpha)}^{L d *}, \quad n_{i j(\alpha)}^{L L\left(B^{+}, \pi^{+}\right)}=f_{1 i(\alpha)}^{L d} f_{3 j(\alpha)}^{L d *}, \\
& n_{i j(\alpha)}^{L L\left(B^{+}, K^{+}\right)}=f_{2 i(\alpha)}^{L d} f_{3 j(\alpha)}^{L d *}, \quad n_{i j(\alpha)}^{R R}=n_{i j(\alpha)}^{L L}(L \leftrightarrow R), \\
& p_{i j(\alpha)}^{L R\left(K^{+}, \pi^{+}\right)}=\frac{f_{1 i(\alpha)}^{L d} f_{2 j(\alpha)}^{R d *} Q}{m_{s}-m_{d}}, \quad p_{i j(\alpha)}^{L R\left(B^{+}, \pi^{+}\right)}=\frac{f_{1 i(\alpha)}^{L d} f_{3 j(\alpha)}^{R d *} Q}{m_{b}-m_{d}}, \quad p_{i j(\alpha)}^{L R\left(B^{+}, K^{+}\right)}=\frac{f_{2 i(\alpha)}^{L d} f_{3 j(\alpha)}^{R d *} Q}{m_{b}-m_{s}}, \quad p_{i j(\alpha)}^{R L}=p_{i j(\alpha)}^{L R}(L \leftrightarrow R) .
\end{aligned}
$$

The form factors $f_{+}\left(q^{2}\right)$ and $f_{0}\left(q^{2}\right)$ are calculated using lattice methods. For the $K \rightarrow \pi$ form factor we use the linear fit given in [77]. For the $B \rightarrow \pi$ and $B \rightarrow K$ form factors we adopt the results of [28,78], where the interpolating functions were obtained using the Bourrely-Caprini-Lellouch parametrization [61].

The $K \rightarrow \pi$ form factor is [77]

$$
f_{\{+, 0\}}\left(q^{2}\right)_{K \pi}=f_{+}(0)_{K \pi}\left[1+\lambda_{\{+, 0\}}^{\prime} \frac{q^{2}}{m_{\pi^{+}}^{2}}\right],
$$

with $f_{+}(0)_{K \pi}=0.9636, \lambda_{+}^{\prime}=0.0308$ and $\lambda_{0}^{\prime}=0.0198$.

The $B \rightarrow \pi$ and $B \rightarrow K$ form factors are given by

$$
f_{+}\left(q^{2}\right)=\frac{1}{P_{+}\left(q^{2}\right)} \sum_{n=0}^{N_{+}-1} b_{+}^{(n)}\left[z^{n}-(-1)^{n-N_{+}} \frac{n}{N_{+}} z^{N_{+}}\right], \quad f_{0}\left(q^{2}\right)=\sum_{n=0}^{N_{0}} b_{0}^{(n)} z^{n},
$$

where

$$
z \equiv z\left(q^{2}\right)=\frac{\sqrt{t_{+}-q^{2}}-\sqrt{t_{+}-t_{0}}}{\sqrt{t_{+}-q^{2}}+\sqrt{t_{+}-t_{0}}} .
$$

In the $B \rightarrow \pi$ case [78], 
$t_{+}=\left(m_{B^{+}}+m_{\pi^{+}}\right)^{2}, t_{-}=\left(m_{B^{+}}-m_{\pi^{+}}\right)^{2}$,

$t_{0}=\left(m_{B^{+}}+m_{\pi^{+}}\right)\left(\sqrt{m_{B^{+}}}-\sqrt{m_{\pi^{+}}}\right)^{2}$,

$b_{+}^{(0)}=0.42, b_{+}^{(1)}=-1.46 b_{+}^{(0)}, b_{+}^{(2)}=-4.7 b_{+}^{(0)}$,

$b_{0}^{(0)}=0.516, b_{0}^{(1)}=-3.94 b_{0}^{(0)}, b_{0}^{(2)}=0.7 b_{0}^{(0)}$,

$P_{+}\left(q^{2}\right)=1-q^{2} / m_{B^{*}}^{2}, m_{B^{*}}=5.325 \mathrm{GeV}$,

whereas for $B \rightarrow K$ [28]

$t_{+}=\left(m_{B^{+}}+m_{K^{+}}\right)^{2}, t_{-}=\left(m_{B^{+}}-m_{K^{+}}\right)^{2}$,

$t_{0}=\left(m_{B^{+}}+m_{K^{+}}\right)\left(\sqrt{m_{B^{+}}}-\sqrt{m_{K^{+}}}\right)^{2}$, $b_{+}^{(0)}=0.432, b_{+}^{(1)}=-0.65, b_{+}^{(2)}=-0.97$,

$b_{0}^{(0)}=0.550, b_{0}^{(1)}=-1.89, b_{0}^{(2)}=1.98, b_{0}^{(3)}=-0.02$,

$P_{+}\left(q^{2}\right)=1-q^{2} /\left(m_{B^{+}}+\Delta_{+}^{*}\right)^{2}, \Delta_{+}^{*}=0.04578 \mathrm{GeV}$.

The resulting constraints on $B^{+}$decays are much weaker than the corresponding bounds presented in [11]. This is due to the fact that the calculation in [11] assumed $f_{+}\left(q^{2}\right)=f_{0}\left(q^{2}\right)=1$. This assumption for the $B \rightarrow \pi$ and $B \rightarrow K$ form factors is quite far from their actual shape.

\section{a. Charged kaon decays}

Experimental constraints from searches for the processes $K^{+} \rightarrow \pi^{+} e^{ \pm} \mu^{\mp}$ yield

$$
\begin{aligned}
& N_{12}^{+\left(K^{+}, \pi^{+}\right)}+\left(0.54 \mathrm{GeV}^{2}\right) P_{12}^{+\left(K^{+}, \pi^{+}\right)}+(0.83 \mathrm{GeV})\left(R_{12}^{+\left(K^{+}, \pi^{+}\right)}-R_{12}^{-\left(K^{+}, \pi^{+}\right)}\right) \lesssim\left[\frac{\mathrm{Br}\left(K^{+} \rightarrow \pi^{+} e^{+} \mu^{-}\right)}{5.2 \times 10^{-10}}\right](32.1 \mathrm{TeV})^{-4} \\
& N_{21}^{+\left(K^{+}, \pi^{+}\right)}+\left(0.54 \mathrm{GeV}^{2}\right) P_{21}^{+\left(K^{+}, \pi^{+}\right)}-(0.83 \mathrm{GeV})\left(R_{21}^{+\left(K^{+}, \pi^{+}\right)}+R_{21}^{-\left(K^{+}, \pi^{+}\right)}\right) \lesssim\left[\frac{\mathrm{Br}\left(K^{+} \rightarrow \pi^{+} e^{-} \mu^{+}\right)}{1.3 \times 10^{-11}}\right](80.6 \mathrm{TeV})^{-4}
\end{aligned}
$$

\section{b. Charged $B$ meson decays}

The experimental bounds on the decays $B^{+} \rightarrow \pi^{+} e^{ \pm} \mu^{\mp}, B^{+} \rightarrow K^{+} e^{ \pm} \mu^{\mp}$ and $B^{+} \rightarrow K^{+} \mu^{ \pm} \tau^{\mp}$ give

$$
\begin{aligned}
& N_{12}^{+\left(B^{+}, \pi^{+}\right)}+\left(138 \mathrm{GeV}^{2}\right) P_{12}^{+\left(B^{+}, \pi^{+}\right)}+(0.76 \mathrm{GeV})\left(R_{12}^{+\left(B^{+}, \pi^{+}\right)}-R_{12}^{-\left(B^{+}, \pi^{+}\right)}\right) \lesssim\left[\frac{\operatorname{Br}\left(B^{+} \rightarrow \pi^{+} e^{+} \mu^{-}\right)}{9.2 \times 10^{-8}}\right](13.5 \mathrm{TeV})^{-4}, \\
& N_{21}^{+\left(B^{+}, \pi^{+}\right)}+\left(138 \mathrm{GeV}^{2}\right) P_{21}^{+\left(B^{+}, \pi^{+}\right)}-(0.76 \mathrm{GeV})\left(R_{21}^{+\left(B^{+}, \pi^{+}\right)}+R_{21}^{-\left(B^{+}, \pi^{+}\right)}\right) \lesssim\left[\frac{\mathrm{Br}\left(B^{+} \rightarrow \pi^{+} e^{-} \mu^{+}\right)}{9.2 \times 10^{-8}}\right](13.5 \mathrm{TeV})^{-4},
\end{aligned}
$$

$$
N_{12}^{+\left(B^{+}, K^{+}\right)}+\left(109 \mathrm{GeV}^{2}\right) P_{12}^{+\left(B^{+}, K^{+}\right)}+(1.0 \mathrm{GeV})\left(R_{12}^{+\left(B^{+}, K^{+}\right)}-R_{12}^{-\left(B^{+}, K^{+}\right)}\right) \lesssim\left[\frac{\mathrm{Br}\left(B^{+} \rightarrow K^{+} e^{+} \mu^{-}\right)}{9.1 \times 10^{-8}}\right](16.2 \mathrm{TeV})^{-4},
$$

$$
N_{21}^{+\left(B^{+}, K^{+}\right)}+\left(109 \mathrm{GeV}^{2}\right) P_{21}^{+\left(B^{+}, K^{+}\right)}-(1.0 \mathrm{GeV})\left(R_{21}^{+\left(B^{+}, K^{+}\right)}+R_{21}^{-\left(B^{+}, K^{+}\right)}\right) \lesssim\left[\frac{\operatorname{Br}\left(B^{+} \rightarrow K^{+} e^{-} \mu^{+}\right)}{1.3 \times 10^{-7}}\right](14.9 \mathrm{TeV})^{-4},
$$

$$
N_{23}^{+\left(B^{+}, K^{+}\right)}+\left(96 \mathrm{GeV}^{2}\right) P_{23}^{+\left(B^{+}, K^{+}\right)}+(10.3 \mathrm{GeV})\left(R_{23}^{+\left(B^{+}, K^{+}\right)}-1.2 R_{23}^{-\left(B^{+}, K^{+}\right)}\right) \lesssim\left[\frac{\operatorname{Br}\left(B^{+} \rightarrow K^{+} \mu^{+} \tau^{-}\right)}{7.7 \times 10^{-5}}\right](2.7 \mathrm{TeV})^{-4},
$$

$$
N_{32}^{+\left(B^{+}, K^{+}\right)}+\left(96 \mathrm{GeV}^{2}\right) P_{32}^{+\left(B^{+}, K^{+}\right)}-(10.3 \mathrm{GeV})\left(R_{23}^{+\left(B^{+}, K^{+}\right)}+1.2 R_{23}^{-\left(B^{+}, K^{+}\right)}\right) \lesssim\left[\frac{\mathrm{Br}\left(B^{+} \rightarrow K^{+} \mu^{-} \tau^{+}\right)}{7.7 \times 10^{-5}}\right](2.7 \mathrm{TeV})^{-4}
$$




\section{Tau decays}

The leptoquark contribution to the rate of $\tau$ decays to a pseudoscalar meson and a lepton, neglecting the mass of the lepton in the final state, is

$$
\Gamma\left(\tau^{-} \rightarrow \mathcal{M}^{\prime} l_{i}^{-}\right)_{X}=\frac{m_{\tau}^{3} f_{\mathcal{M}^{\prime}}^{2}}{128 \pi} T_{i}\left(1-\frac{m_{\mathcal{M}^{\prime}}^{2}}{m_{\tau}^{2}}\right)^{2},
$$

where

$$
\begin{aligned}
T_{i} \equiv & \left|\sum_{\alpha} \frac{t_{i(\alpha)}^{L}}{M_{\alpha}^{2}}\right|^{2}+\left|\sum_{\alpha} \frac{t_{i(\alpha)}^{R}}{M_{\alpha}^{2}}\right|^{2}, \\
t_{i(\alpha)}^{L\left(\pi^{0}\right)} \equiv & f_{13(\alpha)}^{R d} f_{1 i(\alpha)}^{R d *}+\frac{2 m_{\pi^{0}}^{2} Q}{m_{\tau}\left(m_{d}+m_{u}\right)} f_{13(\alpha)}^{L d} f_{1 i(\alpha)}^{R d *}, \\
t_{i(\alpha)}^{L\left(K_{S}^{0}\right)} \equiv & \frac{1}{\sqrt{2}}\left[f_{13(\alpha)}^{R d} f_{2 i(\alpha)}^{R d *}+\frac{2 m_{K_{S}^{0}}^{2} Q}{m_{\tau}\left(m_{s}+m_{d}\right)} f_{13(\alpha)}^{L d} f_{2 i(\alpha)}^{R d *}\right] \\
& -(1 \leftrightarrow 2), \\
t_{i(\alpha)}^{R} \equiv & t_{i(\alpha)}^{L}(L \leftrightarrow R)
\end{aligned}
$$

and $f_{\pi^{0}}=f_{\pi^{+}} / \sqrt{2}$. The bounds are

$$
\begin{aligned}
T_{1}^{\left(\pi^{0}\right)} \lesssim\left[\frac{\operatorname{Br}\left(\tau^{-} \rightarrow \pi^{0} e^{-}\right)}{8.0 \times 10^{-8}}\right](5.0 \mathrm{TeV})^{-4}, \\
T_{2}^{\left(\pi^{0}\right)} \lesssim\left[\frac{\operatorname{Br}\left(\tau^{-} \rightarrow \pi^{0} \mu^{-}\right)}{1.1 \times 10^{-7}}\right](4.7 \mathrm{TeV})^{-4},
\end{aligned}
$$

$T_{1}^{\left(K_{S}^{0}\right)} \lesssim\left[\frac{\mathrm{Br}\left(\tau^{-} \rightarrow K_{S}^{0} e^{-}\right)}{2.6 \times 10^{-8}}\right](8.4 \mathrm{TeV})^{-4}$,

$T_{2}^{\left(K_{S}^{0}\right)} \lesssim\left[\frac{\mathrm{Br}\left(\tau^{-} \rightarrow K_{S}^{0} \mu^{-}\right)}{2.3 \times 10^{-8}}\right](8.6 \mathrm{TeV})^{-4}$.

There are also constraints from $\tau$ decays to a vector meson and a lepton, e.g., $\tau^{-} \rightarrow \phi l_{i}^{-}$. The resulting leptoquark contribution to the rate is given by Eq. (C48) with
$t_{i(\alpha)}^{L(\phi)} \equiv \sqrt{1+\frac{2 m_{\phi}^{2}}{m_{\tau}^{2}}}\left(f_{23(\alpha)}^{R d} f_{2 i(\alpha)}^{R d *}+\frac{m_{\phi}^{2} Q}{m_{\tau} m_{s}} f_{23(\alpha)}^{L d} f_{2 i(\alpha)}^{R d *}\right)$,

$t_{i(\alpha)}^{R(\phi)} \equiv t_{i(\alpha)}^{L(\phi)}(L \leftrightarrow R)$.

The experimental bounds yield

$T_{1}^{(\phi)} \lesssim\left[\frac{\operatorname{Br}\left(\tau^{-} \rightarrow \phi e^{-}\right)}{3.1 \times 10^{-8}}\right](9.6 \mathrm{TeV})^{-4}$

$T_{2}^{(\phi)} \lesssim\left[\frac{\operatorname{Br}\left(\tau^{-} \rightarrow \phi \mu^{-}\right)}{8.4 \times 10^{-8}}\right](7.5 \mathrm{TeV})^{-4}$

\section{Radiative charged lepton decay}

The vector leptoquark contribution to the process $l_{i} \rightarrow l_{j} \gamma$ is induced at the loop level. Unlike for scalar leptoquarks, in the case of vector leptoquarks this effect cannot be computed in the general case, since the result is infinite and requires arbitrary subtractions that are well defined only in a UV complete model. We parametrize our ignorance of this UV completion with the coefficients $c_{L R}$ and $c_{R L}$,

$$
\begin{aligned}
\Gamma\left(l_{i}^{+} \rightarrow l_{j}^{+} \gamma\right)_{X}= & \frac{e^{2} m_{i}^{5}}{4096 \pi^{5}}\left[\left|\sum_{\alpha ; k} \frac{f_{k j(\alpha)}^{L d} f_{k i(\alpha)}^{L d *}}{M_{\alpha}^{2}}\right|^{2}+(L \leftrightarrow R)\right] \\
& +\frac{e^{2} m_{i}^{3} m_{b}^{2}}{4096 \pi^{5}}\left[c_{L R}^{2}\left|\sum_{\alpha} \frac{f_{3 j(\alpha)}^{L d} f_{3 i(\alpha)}^{R d *}}{M_{\alpha}^{2}}\right|^{2}\right. \\
& +(L \leftrightarrow R)]+\cdots, \quad(\mathrm{C} 57)
\end{aligned}
$$

where $k=1,2,3$ and we expect $c_{L R}$ and $c_{R L}$ to be $\mathcal{O}(1)$, with their values dependent on the UV details of the model. The ellipsis denotes interference and mass-suppressed terms.

If the matrices $f_{i j}$ are proportional to unitary matrices, the terms in the first line of Eq. (C57) vanish. The experimental bounds, neglecting higher order terms, become

$$
\begin{aligned}
& c_{L R}^{2}\left|\sum_{\alpha} \frac{f_{31(\alpha)}^{L d} f_{32(\alpha)}^{R d *}}{M_{\alpha}^{2}}\right|^{2}+c_{R L}^{2}\left|\sum_{\alpha} \frac{f_{31(\alpha)}^{R d} f_{32(\alpha)}^{L d *}}{M_{\alpha}^{2}}\right|^{2} \lesssim\left[\frac{\mathrm{Br}(\mu \rightarrow e \gamma)}{4.2 \times 10^{-13}}\right](332 \mathrm{TeV})^{-4}, \\
& c_{L R}^{2}\left|\sum_{\alpha} \frac{f_{31(\alpha)}^{L d} f_{33(\alpha)}^{R d *}}{M_{\alpha}^{2}}\right|^{2}+c_{R L}^{2}\left|\sum_{\alpha} \frac{f_{31(\alpha)}^{R d} f_{33(\alpha)}^{L d *}}{M_{\alpha}^{2}}\right|^{2} \lesssim\left[\frac{\mathrm{Br}(\tau \rightarrow e \gamma)}{3.3 \times 10^{-8}}\right](3.1 \mathrm{TeV})^{-4}, \\
& c_{L R}^{2}\left|\sum_{\alpha} \frac{f_{32(\alpha)}^{L d} f_{33(\alpha)}^{R d *}}{M_{\alpha}^{2}}\right|^{2}+c_{R L}^{2}\left|\sum_{\alpha} \frac{f_{32(\alpha)}^{R d} f_{33(\alpha)}^{L d *}}{M_{\alpha}^{2}}\right|^{2} \lesssim\left[\frac{\mathrm{Br}(\tau \rightarrow \mu \gamma)}{4.4 \times 10^{-8}}\right](2.9 \mathrm{TeV})^{-4} .
\end{aligned}
$$


In our model the leading order terms contributing to $l_{i}^{+} \rightarrow l_{j}^{+} \gamma$ are $\mathcal{O}\left(m_{b}^{2} / M_{X_{L}}^{2}\right)$ and the resulting constraints are negligible compared to tree-level bounds.

\section{6. $l_{i}^{+} \rightarrow l_{j}^{+}$conversion}

The effective Hamiltonian for the $l_{i}^{+} \rightarrow l_{j}^{+}$conversion consists of the dipole transition part corresponding to $l_{i}^{+} \rightarrow l_{j}^{+} \gamma$ and terms arising from integrating out the heavy vector leptoquarks, i.e.,

$$
\begin{aligned}
\mathcal{H}_{l_{i}^{+} \rightarrow l_{j}^{+}}^{\mathrm{eff}}= & \frac{e m_{b}}{16 \pi^{2}} \sum_{\alpha} \frac{c_{L R}}{M_{\alpha}^{2}} f_{3 j(\alpha)}^{L d} f_{3 i(\alpha)}^{R d *} \bar{l}_{j R} \sigma_{\mu \nu} l_{i L} F^{\mu \nu} \\
& +\frac{e m_{i}}{16 \pi^{2}} \sum_{\alpha, k} \frac{1}{M_{\alpha}^{2}} f_{k j(\alpha)}^{L d} f_{k i(\alpha)}^{L d *} \bar{l}_{j R} \sigma_{\mu \nu} l_{i L} F^{\mu \nu} \\
& +\sum_{\alpha, m} \frac{1}{M_{\alpha}^{2}}\left[f_{m j(\alpha)}^{L d} f_{m i(\alpha)}^{L d *}\left(\bar{l}_{j L} \gamma^{\mu} l_{i L}\right)\left(\bar{d}_{L}^{k} \gamma_{\mu} d_{L}^{k}\right)\right. \\
& \left.-2 Q f_{m j(\alpha)}^{L d} f_{m i(\alpha)}^{R d *}\left(\bar{l}_{j L} l_{i R}\right)\left(\bar{d}_{R}^{k} d_{L}^{k}\right)\right] \\
& +(L \leftrightarrow R)+\cdots,
\end{aligned}
$$

where $m=1,2$. The steps required to match the effective Hamiltonian (C61) to the Hamiltonian at the nucleon level and compute the conversion rate are provided in [81,82].

The tightest experimental constraint from $l_{i}^{+}-l_{j}^{+}$conversion arises from $\mu-e$ conversion on gold [59]. Since the resulting bound on the dipole transition contribution is less restrictive than the constraint from $\mu \rightarrow e \gamma$ in Eq. (C58), we concentrate only on the second part of the Hamiltonian (C61). Following [81], the $\mu-e$ conversion rate is then given by

$$
\begin{aligned}
\Gamma(\mu \rightarrow e)= & m_{\mu}^{5}\left|\tilde{g}_{L V}^{(p)} V_{p}+\tilde{g}_{L V}^{(n)} V_{n}+\tilde{g}_{L S}^{(p)} S_{p}+\tilde{g}_{L S}^{(n)} S_{n}\right|^{2} \\
& +(L \leftrightarrow R),
\end{aligned}
$$

where

$$
\begin{aligned}
& \tilde{g}_{L V}^{(p)}=\frac{1}{2} \tilde{g}_{L V}^{(n)}=\sum_{\alpha} \frac{1}{M_{\alpha}^{2}} f_{12(\alpha)}^{L d} f_{11(\alpha)}^{L d *}, \\
& \tilde{g}_{L S}^{(p)}=-2 Q \sum_{\alpha} \frac{1}{M_{\alpha}^{2}}\left[4.3 f_{12(\alpha)}^{L d} f_{11(\alpha)}^{R d *}+2.5 f_{22(\alpha)}^{L d} f_{21(\alpha)}^{R d *}\right], \\
& \tilde{g}_{L S}^{(n)}=-2 Q \sum_{\alpha} \frac{1}{M_{\alpha}^{2}}\left[5.1 f_{12(\alpha)}^{L d} f_{11(\alpha)}^{R d *}+2.5 f_{22(\alpha)}^{L d} f_{21(\alpha)}^{R d *}\right],
\end{aligned}
$$

with similar relations obtained upon switching $(L \leftrightarrow R)$. The numerical coefficients were adopted from [83]. For the ${ }_{79}^{197} \mathrm{Au}$ nucleus, which provides the most stringent bound, the parameters in Eq. (C62) are

$$
V_{p}=0.0974, \quad V_{n}=0.146, \quad S_{p}=0.0614, \quad S_{n}=0.0918,
$$

and they are the result of the calculation using "method 1 " in Sec. III A of [81]. The best bound on $\mu-e$ conversion is [59]

$$
\frac{\Gamma(\mu \rightarrow e \text { in } \mathrm{Au})}{\Gamma(\mu \text { capture in } \mathrm{Au})}<7 \times 10^{-13} .
$$

The constraints on general $(3,1)_{2 / 3}$ leptoquark models are derived by inserting Eq. (C62) into (C64) and using the total $\mu^{-}$capture rate in ${ }_{79}^{197} \mathrm{Au}, \Gamma(\mu$ capture in $\mathrm{Au})=8.6 \times$ $10^{-18} \mathrm{GeV}$ [84]. In the case of our model, with just LH leptoquark couplings, the constraint simplifies to

$$
\left|\sum_{\alpha} \frac{f_{12(\alpha)}^{L d} f_{11(\alpha)}^{L d *}}{M_{\alpha}^{2}}\right|^{-1 / 2} \gtrsim 762 \mathrm{TeV} .
$$

Finally, let us note that the bounds on generic leptoquark models were considered in [7-12]. Our formulas reproduce those results up to the difference in the adopted values of quark masses, meson decay constants and form factors used.

\section{APPENDIX D: FLAVOR CONSTRAINTS: $\mathrm{SU}(4)_{L} \times \mathrm{SU}(4)_{L}$ MODEL}

In our model $X^{(1)} \equiv X_{1}$ and $X^{(2)} \equiv X_{2}$ given by Eq. (15); therefore the coefficients in Eq. (C1) are

$$
\begin{array}{rlrl}
f_{i j(1)}^{L u} & \equiv \frac{g_{L} \cos \theta_{4}}{\sqrt{2}} L_{i j}^{u}, & f_{i j(1)}^{L d} \equiv \frac{g_{L} \cos \theta_{4}}{\sqrt{2}} L_{i j}^{d}, \\
f_{i j(1)}^{R u} \equiv \frac{g_{R} \sin \theta_{4}}{\sqrt{2}} R_{i j}^{u}, & f_{i j(1)}^{R d} \equiv \frac{g_{R} \sin \theta_{4}}{\sqrt{2}} R_{i j}^{d}, \\
f_{i j(2)}^{R u} \equiv \frac{g_{R} \cos \theta_{4}}{\sqrt{2}} R_{i j}^{u}, & f_{i j(2)}^{R d} \equiv \frac{g_{R} \cos \theta_{4}}{\sqrt{2}} R_{i j}^{d}, \\
f_{i j(2)}^{L u} \equiv-\frac{g_{L} \sin \theta_{4}}{\sqrt{2}} L_{i j}^{u}, & f_{i j(2)}^{L d} \equiv-\frac{g_{L} \sin \theta_{4}}{\sqrt{2}} L_{i j}^{d} .
\end{array}
$$

Constraints on the model parameters are obtained by substituting the expressions in Eq. (D1) into the bounds derived in Appendix C. In the limit $v_{R} \gg v_{L}$ and $v_{R} \gg v_{\Sigma}$, for which $\sin \theta_{4} \simeq 0, X_{1}=X_{L}$ and $X_{2}=X_{R}$, one arrives at the constraints listed below. It is explicitly shown which equation in Appendix $\mathrm{C}$ a given constraint originated from.

\section{1. $K_{L}^{0}$ decays}

$$
\begin{gathered}
(\mathrm{C} 13): \frac{M_{X_{L}}}{g_{L} \sqrt{\left|\operatorname{Re}\left(L_{11}^{d} L_{21}^{d *}\right)\right|}} \gtrsim 21.2 \mathrm{TeV}, \\
(\mathrm{C} 14): \frac{M_{X_{L}}}{g_{L} \sqrt{\left|L_{11}^{d} L_{22}^{d *}+L_{21}^{d} L_{12}^{d *}\right|}} \gtrsim 225 \mathrm{TeV}, \\
(\mathrm{C} 15): \frac{M_{X_{L}}}{g_{L} \sqrt{\left|\operatorname{Re}\left(L_{12}^{d} L_{22}^{d *}\right)\right|}} \gtrsim 51.0 \mathrm{TeV} .
\end{gathered}
$$


2. $B^{\mathbf{0}}$ decays
$(\mathrm{C} 16): \frac{M_{X_{L}}}{g_{L} \sqrt{\left|L_{11}^{d} L_{31}^{d *}\right|}} \gtrsim 0.24 \mathrm{TeV}$,

$(\mathrm{C} 17): \frac{M_{X_{L}}}{g_{L} \sqrt[4]{\left|L_{11}^{d} L_{32}^{d *}\right|^{2}+\left|L_{12}^{d} L_{31}^{d *}\right|^{2}}} \gtrsim 8.9 \mathrm{TeV}$,

$(\mathrm{C} 18): \frac{M_{X_{L}}}{g_{L} \sqrt{\left|L_{12}^{d} L_{32}^{d *}\right|}} \gtrsim 10.7 \mathrm{TeV}$

$(\mathrm{C} 19): \frac{M_{X_{L}}}{g_{L} \sqrt[4]{\left|L_{11}^{d} L_{33}^{d *}\right|^{2}+\left|L_{13}^{d} L_{31}^{d *}\right|^{2}}} \gtrsim 2.6 \mathrm{TeV}$,

$(\mathrm{C} 20): \frac{M_{X_{L}}}{g_{L} \sqrt[4]{\left|L_{12}^{d} L_{33}^{d *}\right|^{2}+\left|L_{13}^{d} L_{32}^{d *}\right|^{2}}} \gtrsim 2.8 \mathrm{TeV}$,

$(\mathrm{C} 21): \frac{M_{X_{L}}}{g_{L} \sqrt{\left|L_{13}^{d} L_{33}^{d *}\right|}} \gtrsim 1.0 \mathrm{TeV}$.

\section{3. $B_{s}^{0}$ decays}

$(\mathrm{C} 22): \frac{M_{X_{L}}}{g_{L} \sqrt{\left|L_{21}^{d} L_{31}^{d *}\right|}} \gtrsim 0.2 \mathrm{TeV}$,

$(\mathrm{C} 23): \frac{M_{X_{L}}}{g_{L} \sqrt[4]{\left|L_{21}^{d} L_{32}^{d *}\right|^{2}+\left|L_{22}^{d} L_{31}^{d *}\right|^{2}}} \gtrsim 6.4 \mathrm{TeV}$,

$(\mathrm{C} 24): \frac{M_{X_{L}}}{g_{L} \sqrt{\left|L_{22}^{d} L_{32}^{d *}\right|}} \gtrsim 6.0 \mathrm{TeV}$,

$(\mathrm{C} 25): \frac{M_{X_{L}}}{g_{L} \sqrt{\left|L_{23}^{d} L_{33}^{d *}\right|}} \gtrsim 0.7 \mathrm{TeV}$.

(D14)

\section{4. $\pi^{+}$decays}

$(\mathrm{C} 32): \frac{M_{X_{L}}}{g_{L} \sqrt{\left|\operatorname{Re}\left[L_{11}^{d}\left(V L^{d}\right)_{11}^{*}-4.3 L_{12}^{d}\left(V L^{d}\right)_{12}^{*}\right]\right|}} \gtrsim 2.8 \mathrm{TeV}$.

(D15)

\section{5. $\mathrm{K}^{+}$decays}

(C33): $\frac{M_{X_{L}}}{g_{L} \sqrt{\left|\operatorname{Re}\left[L_{11}^{d}\left(V L^{d}\right)_{21}^{*}-4.3 L_{12}^{d}\left(V L^{d}\right)_{22}^{*}\right]\right|}} \gtrsim 2.2 \mathrm{TeV}$,

$(\mathrm{C} 41): \frac{M_{X_{L}}}{g_{L} \sqrt{\left|L_{12}^{d} L_{21}^{d *}\right|}} \gtrsim 67.8 \mathrm{TeV}$.

\section{6. $B^{+}$decays}

$(\mathrm{C} 42): \frac{M_{X_{L}}}{g_{L} \sqrt{\left|L_{11}^{d} L_{32}^{d *}\right|}} \gtrsim 11.4 \mathrm{TeV}$,

$(\mathrm{C} 43): \frac{M_{X_{L}}}{g_{L} \sqrt{\left|L_{12}^{d} L_{31}^{d *}\right|}} \gtrsim 11.4 \mathrm{TeV}$,

$(\mathrm{C} 44): \frac{M_{X_{L}}}{g_{L} \sqrt{\left|L_{21}^{d} L_{32}^{d *}\right|}} \gtrsim 13.6 \mathrm{TeV}$,

$(\mathrm{C} 45): \frac{M_{X_{L}}}{g_{L} \sqrt{\left|L_{22}^{d} L_{31}^{d *}\right|}} \gtrsim 12.5 \mathrm{TeV}$,

(C46): $\frac{M_{X_{L}}}{g_{L} \sqrt{\left|L_{22}^{d} L_{33}^{d *}\right|}} \gtrsim 2.3 \mathrm{TeV}$,

(C47): $\frac{M_{X_{L}}}{g_{L} \sqrt{\left|L_{23}^{d} L_{32}^{d *}\right|}} \gtrsim 2.3 \mathrm{TeV}$.

\section{7. $\tau$ decays}

$(\mathrm{C} 50): \frac{M_{X_{L}}}{g_{L} \sqrt{\left|L_{11}^{d} L_{13}^{d *}\right|}} \gtrsim 3.6 \mathrm{TeV}$,

(C51): $\frac{M_{X_{L}}}{g_{L} \sqrt{\left|L_{12}^{d} L_{13}^{d *}\right|}} \gtrsim 3.3 \mathrm{TeV}$,

$(\mathrm{C} 52): \frac{M_{X_{L}}}{g_{L} \sqrt{\left|L_{21}^{d} L_{13}^{d *}-L_{11}^{d} L_{23}^{d *}\right|}} \gtrsim 5.0 \mathrm{TeV}$, 


$$
\begin{gathered}
(\mathrm{C} 53): \frac{M_{X_{L}}}{g_{L} \sqrt{\left|L_{22}^{d} L_{13}^{d *}-L_{12}^{d} L_{23}^{d *}\right|}} \gtrsim 5.1 \mathrm{TeV}, \\
(\mathrm{C} 55): \frac{M_{X_{L}}}{g_{L} \sqrt{\left|L_{21}^{d} L_{23}^{d *}\right|}} \gtrsim 6.8 \mathrm{TeV},
\end{gathered}
$$

$$
(\mathrm{C} 56): \frac{M_{X_{L}}}{g_{L} \sqrt{\left|L_{22}^{d} L_{23}^{d *}\right|}} \gtrsim 5.3 \mathrm{TeV}
$$

\section{8. $\mu-e$ conversion}

$(\mathrm{C} 65): \frac{M_{X_{L}}}{g_{L} \sqrt{\left|L_{12}^{d} L_{11}^{d *}\right|}} \gtrsim 539 \mathrm{TeV}$.
[1] R. Aaij et al. (LHCb Collaboration), Test of Lepton Universality Using $\mathrm{B}^{+} \rightarrow \mathrm{K}^{+} \ell^{+} \ell^{-}$Decays, Phys. Rev. Lett. 113, 151601 (2014).

[2] R. Aaij et al. (LHCb Collaboration), Test of lepton universality with $B^{0} \rightarrow K^{* 0} \ell^{+} \ell^{-}$decays, J. High Energy Phys. 08 (2017) 055.

[3] R. Alonso, B. Grinstein, and J. Martin Camalich, Lepton universality violation and lepton flavor conservation in $B$ meson decays, J. High Energy Phys. 10 (2015) 184.

[4] R. Alonso, B. Grinstein, and J. Martin Camalich, $S U(2) \times$ $U(1)$ Gauge Invariance and the Shape of New Physics in Rare B Decays, Phys. Rev. Lett. 113, 241802 (2014).

[5] N. Kosnik, Model independent constraints on leptoquarks from $b \rightarrow s \ell^{+} \ell^{-}$processes, Phys. Rev. D 86, 055004 (2012).

[6] N. Assad, B. Fornal, and B. Grinstein, Baryon number and lepton universality violation in leptoquark and diquark models, Phys. Lett. B 777, 324 (2018).

[7] G. Valencia and S. Willenbrock, Quark-lepton unification and rare meson decays, Phys. Rev. D 50, 6843 (1994).

[8] A. D. Smirnov, Mass limits for scalar and gauge leptoquarks from $K_{L}^{0} \rightarrow e^{\mp} \mu^{ \pm}, B^{0} \rightarrow e^{\mp} \tau^{ \pm}$decays, Mod. Phys. Lett. A 22, 2353 (2007).

[9] A. D. Smirnov, Contributions of gauge and scalar leptoquarks to $K_{L}^{0} \rightarrow l_{i}^{+} l_{j}^{-}$and $B^{0} \rightarrow l_{i}^{+} l_{j}^{-}$decay and constraints on leptoquark masses from the decays $K_{L}^{0} \rightarrow e^{\mp} \mu^{ \pm}$and $B^{0} \rightarrow e^{\mp} \tau^{ \pm}$, Phys. At. Nucl. 71, 1470 (2008).

[10] M. Carpentier and S. Davidson, Constraints on two-lepton, two quark operators, Eur. Phys. J. C 70, 1071 (2010).

[11] A. V. Kuznetsov, N. V. Mikheev, and A. V. Serghienko, The third type of fermion mixing in the lepton and quark interactions with leptoquarks, Int. J. Mod. Phys. A 27, 1250062 (2012).

[12] A. D. Smirnov, Vector leptoquark mass limits and branching ratios of $K_{L}^{0}, B^{0}, B_{s} \rightarrow l_{i}^{+} l_{j}^{-}$decays with account of fermion mixing in leptoquark currents, Mod. Phys. Lett. A 33, 1850019 (2018).

[13] L. Di Luzio, A. Greljo, and M. Nardecchia, Gauge leptoquark as the origin of $B$-physics anomalies, Phys. Rev. D 96, 115011 (2017).

[14] L. Calibbi, A. Crivellin, and T. Li, A model of vector leptoquarks in view of the $B$-physics anomalies, Phys. Rev. D 98, 115002 (2018).
[15] M. Bordone, C. Cornella, J. Fuentes-Martin, and G. Isidori, A three-site gauge model for flavor hierarchies and flavor anomalies, Phys. Lett. B 779, 317 (2018).

[16] R. Barbieri and A. Tesi, B-decay anomalies in Pati-Salam SU(4), Eur. Phys. J. C 78, 193 (2018).

[17] M. Blanke and A. Crivellin, $B$ Meson Anomalies in a Pati-Salam Model within the Randall-Sundrum Background, Phys. Rev. Lett. 121, 011801 (2018).

[18] A. Greljo and B. A. Stefanek, Third family quarklepton unification at the TeV scale, Phys. Lett. B 782, 131 (2018).

[19] S. Balaji, R. Foot, and M. A. Schmidt, A chiral SU(4) explanation of the $b \rightarrow s$ anomalies, Phys. Rev. D 99, 015029 (2019).

[20] D. Marzocca, Addressing the $B$-physics anomalies in a fundamental composite Higgs model, J. High Energy Phys. 07 (2018) 121.

[21] U. Aydemir, D. Minic, C. Sun, and T. Takeuchi, B-decay anomalies and scalar leptoquarks in unified Pati-Salam models from noncommutative geometry, J. High Energy Phys. 09 (2018) 117.

[22] D. Becirevic, I. Dorsner, S. Fajfer, D. A. Faroughy, N. Kosnik, and O. Sumensari, Scalar leptoquarks from GUT to accommodate the $B$-physics anomalies, Phys. Rev. D 98, 055003 (2018).

[23] D. Guadagnoli, M. Reboud, and O. Sumensari, A gauged horizontal $S U(2)$ symmetry and $R_{K^{(*)}}$, J. High Energy Phys. 11 (2018) 163.

[24] S.-P. Li, X.-Q. Li, Y.-D. Yang, and X. Zhang, $R_{D^{(*)}}, R_{K^{(*)}}$ and neutrino mass in the 2HDM-III with right-handed neutrinos, J. High Energy Phys. 09 (2018) 149.

[25] T. Faber, M. Hudec, M. Malinsky, P. Meinzinger, W. Porod, and F. Staub, A unified leptoquark model confronted with lepton non-universality in $B$-meson decays, Phys. Lett. B 787, 159 (2018).

[26] J. Heeck and D. Teresi, Pati-Salam explanations of the $B$ meson anomalies, J. High Energy Phys. 12 (2018) 103.

[27] B. Allanach and J. Davighi, Third family hypercharge model for $R_{K^{(*)}}$ and aspects of the Fermion mass problem, J. High Energy Phys. 12 (2018) 075.

[28] C. Bouchard, G. P. Lepage, C. Monahan, H. Na, and J. Shigemitsu (HPQCD Collaboration), Rare decay $B \rightarrow$ $K \ell^{+} \ell^{-}$form factors from lattice QCD, Phys. Rev. D 88, 
054509 (2013); Erratum, Phys. Rev. D88, 079901(E) (2013).

[29] J. M. Arnold, B. Fornal, and M. B. Wise, Simplified models with Baryon number violation but no proton decay, Phys. Rev. D 87, 075004 (2013).

[30] L.-S. Geng, B. Grinstein, S. Jager, J. Martin Camalich, X.-L. Ren, and R.-X. Shi, Towards the discovery of new physics with lepton-universality ratios of $b \rightarrow s \ell \ell$ decays, Phys. Rev. D 96, 093006 (2017).

[31] M. Ciuchini, A. M. Coutinho, M. Fedele, E. Franco, A. Paul, L. Silvestrini, and M. Valli, On flavourful Easter eggs for new physics hunger and lepton flavour universality violation, Eur. Phys. J. C 77, 688 (2017).

[32] G. Hiller and I. Nisandzic, $R_{K}$ and $R_{K^{*}}$ beyond the standard model, Phys. Rev. D 96, 035003 (2017).

[33] G. D’Amico, M. Nardecchia, P. Panci, F. Sannino, A. Strumia, R. Torre, and A. Urbano, Flavour anomalies after the $R_{K^{*}}$ measurement, J. High Energy Phys. 09 (2017) 010.

[34] W. Altmannshofer, P. Stangl, and D. M. Straub, Interpreting hints for lepton flavor universality violation, Phys. Rev. D 96, 055008 (2017).

[35] B. Capdevila, A. Crivellin, S. Descotes-Genon, J. Matias, and J. Virto, Patterns of new physics in $b \rightarrow s \ell^{+} \ell^{-}$ transitions in the light of recent data, J. High Energy Phys. 01 (2018) 093.

[36] A. Celis, J. Fuentes-Martin, A. Vicente, and J. Virto, Gaugeinvariant implications of the LHCb measurements on lepton-flavor nonuniversality, Phys. Rev. D 96, 035026 (2017).

[37] A. K. Alok, B. Bhattacharya, A. Datta, D. Kumar, J. Kumar, and D. London, New physics in $b \rightarrow s \mu^{+} \mu^{-}$after the measurement of $R_{K^{*}}$, Phys. Rev. D 96, 095009 (2017).

[38] D. I. Britton et al., Measurement of the $\pi^{+} \rightarrow e^{+} \nu$ Branching Ratio, Phys. Rev. Lett. 68, 3000 (1992).

[39] D. I. Britton et al., Measurement of the $\pi^{+} \rightarrow e^{+} \nu$ branching ratio, Phys. Rev. D 49, 28 (1994).

[40] G. Czapek et al., Branching Ratio for the Rare Pion Decay into Positron and Neutrino, Phys. Rev. Lett. 70, 17 (1993).

[41] D. Ambrose et al. (BNL and E871 Collaborations), First Observation of the Rare Decay Mode $K_{L}^{0} \rightarrow e^{+} e^{-}$, Phys. Rev. Lett. 81, 4309 (1998).

[42] D. Ambrose et al. (BNL Collaboration), New Limit on Muon and Electron Lepton Number Violation from $K_{L}^{0} \rightarrow$ $\mu^{ \pm} e^{\mp}$ Decay, Phys. Rev. Lett. 81, 5734 (1998).

[43] D. Ambrose et al. (E871 Collaboration), Improved Branching Ratio Measurement for the Decay $K_{L}^{0} \rightarrow \mu^{+} \mu^{-}$, Phys. Rev. Lett. 84, 1389 (2000).

[44] R. Appel et al., Search for Lepton Flavor Violation in $\mathrm{K}^{+}$ Decays, Phys. Rev. Lett. 85, 2877 (2000).

[45] A. Sher et al., An improved upper limit on the decay $K^{+} \rightarrow \pi^{+} \mu^{+} e^{-}$, Phys. Rev. D 72, 012005 (2005).

[46] F. Ambrosino et al. (KLOE Collaboration), Precise measurement of $\Gamma(K \rightarrow e \nu(\gamma)) / \Gamma(K \rightarrow \mu \nu(\gamma))$ and study of $K \rightarrow e \nu \gamma$, Eur. Phys. J. C 64, 627 (2009); Erratum, Eur. Phys. J. C65, 703(E) (2010).

[47] B. Aubert et al. (BABAR Collaboration), Measurements of branching fractions, rate asymmetries, and angular distributions in the rare decays $B \rightarrow K \ell^{+} \ell^{-}$and $B \rightarrow K^{*} \ell^{+} \ell^{-}$, Phys. Rev. D 73, 092001 (2006).
[48] B. Aubert et al. (BABAR Collaboration), Search for the Rare Decay $B \rightarrow \pi l^{+} l^{-}$, Phys. Rev. Lett. 99, 051801 (2007).

[49] B. Aubert et al. (BABAR Collaboration), Search for the Decay $B^{+} \rightarrow K^{+} \tau^{\mp} \mu^{ \pm}$, Phys. Rev. Lett. 99, 201801 (2007).

[50] B. Aubert et al. (BABAR Collaboration), Searches for the decays $B^{0} \rightarrow l^{ \pm} \tau^{\mp}$ and $B^{+} \rightarrow l^{+} \nu(l=e, \mu)$ using hadronic tag reconstruction, Phys. Rev. D 77, 091104 (2008).

[51] T. Aaltonen et al. (CDF Collaboration), Search for the Decays $B_{s}^{0} \rightarrow e^{+} \mu^{-}$and $B_{s}^{0} \rightarrow e^{+} e^{-}$in CDF Run II, Phys. Rev. Lett. 102, 201801 (2009).

[52] R. Aaij et al. (LHCb Collaboration), Search for the leptonflavour violating decays $B_{s}^{0} \rightarrow e^{ \pm} \mu^{\mp}$, J. High Energy Phys. 03 (2018) 078.

[53] R. Aaij et al. (LHCb Collaboration), Measurement of the $B_{s}^{0} \rightarrow \mu^{+} \mu^{-}$Branching Fraction and Effective Lifetime and Search for $B^{0} \rightarrow \mu^{+} \mu^{-}$Decays, Phys. Rev. Lett. 118, 191801 (2017).

[54] R. Aaij et al. (LHCb Collaboration), Search for the Decays $B_{s}^{0} \rightarrow \tau^{+} \tau^{-}$and $B^{0} \rightarrow \tau^{+} \tau^{-}$, Phys. Rev. Lett. 118, 251802 (2017).

[55] B. Aubert et al. (BABAR Collaboration), Search for Lepton Flavor Violating Decays $\tau^{ \pm} \rightarrow \ell^{ \pm} \pi^{0}, \ell^{ \pm} \eta, \ell^{ \pm} \eta^{\prime}$, Phys. Rev. Lett. 98, 061803 (2007).

[56] Y. Miyazaki et al. (Belle Collaboration), Search for lepton flavor violating $\tau^{-}$decays into $e l l^{-} \eta, \ell^{-} \eta^{\prime}$ and $\ell^{-} \pi^{0}$, Phys. Lett. B 648, 341 (2007).

[57] Y. Miyazaki et al. (Belle Collaboration), Search for lepton flavor violating $\tau^{-}$decays into $\ell^{-} K_{s}^{0}$ and $\ell^{-} K_{s}^{0} K_{s}^{0}$, Phys. Lett. B 692, 4 (2010).

[58] Y. Miyazaki et al. (Belle Collaboration), Search for leptonflavor-violating tau decays into a lepton and a vector meson, Phys. Lett. B 699, 251 (2011).

[59] W. H. Bertl et al. (SINDRUM II Collaboration), A search for muon to electron conversion in muonic gold, Eur. Phys. J. C 47, 337 (2006).

[60] J. M. Arnold, B. Fornal, and M. B. Wise, Phenomenology of scalar leptoquarks, Phys. Rev. D 88, 035009 (2013).

[61] C. Bourrely, I. Caprini, and L. Lellouch, Model-independent description of $B \rightarrow \pi l \nu$ decays and a determination of $\left|V_{u b}\right|$, Phys. Rev. D 79, 013008 (2009); Erratum, Phys. Rev. D82, 099902(E) (2010).

[62] A. Crivellin, C. Greub, F. Saturnino, and D. Muller, Importance of Loop Effects in Explaining the Accumulated Evidence for New Physics in B Decays with a Vector Leptoquark, Phys. Rev. Lett. 122, 011805 (2019).

[63] J. Alwall, M. Herquet, F. Maltoni, O. Mattelaer, and T. Stelzer, MadGraph 5: Going beyond, J. High Energy Phys. 06 (2011) 128.

[64] A. Alloul, N. D. Christensen, C. Degrande, C. Duhr, and B. Fuks, FeynRules 2.0-A complete toolbox for tree-level phenomenology, Comput. Phys. Commun. 185, 2250 (2014).

[65] M. Benedikt and F. Zimmermann, FCC: Colliders at the energy frontier, in Proceedings, 9th International particle accelerator conference (IPAC 2018), Vancouver, Canada (2018), p. 2908.

[66] J. P. Lees et al. (BABAR Collaboration), Measurement of an excess of $\bar{B} \rightarrow D^{(*)} \tau^{-} \bar{\nu}_{\tau}$ decays and implications for charged Higgs bosons, Phys. Rev. D 88, 072012 (2013). 
[67] M. Huschle et al. (Belle Collaboration), Measurement of the branching ratio of $\bar{B} \rightarrow D^{(*)} \tau^{-} \bar{\nu}_{\tau}$ relative to $\bar{B} \rightarrow D^{(*)} \ell^{-} \bar{\nu}_{\ell}$ decays with hadronic tagging at belle, Phys. Rev. D 92, 072014 (2015).

[68] R. Aaij et al. (LHCb Collaboration), Measurement of the Ratio of Branching Fractions $\mathcal{B}\left(\bar{B}^{0} \rightarrow D^{*+} \tau^{-} \bar{\nu}_{\tau}\right) / \mathcal{B}\left(\bar{B}^{0} \rightarrow\right.$ $D^{*+} \mu^{-} \bar{\nu}_{\mu}$ ), Phys. Rev. Lett. 115, 111803 (2015); Erratum, Phys. Rev. Lett.115, 159901(E) (2015).

[69] J. Kumar, D. London, and R. Watanabe, Combined explanations of the $b \rightarrow s \mu^{+} \mu^{-}$and $b \rightarrow c \tau^{-} \bar{\nu}$ anomalies: A general model analysis, Phys. Rev. D 99, 015007 (2019).

[70] D. Becirevic, S. Fajfer, N. Kosnik, and O. Sumensari, Leptoquark model to explain the $B$-physics anomalies, $R_{K}$ and $R_{D}$, Phys. Rev. D 94, 115021 (2016).

[71] M. Tanabashi et al. (Particle Data Group), Review of particle physics, Phys. Rev. D 98, 030001 (2018).

[72] B. Chakraborty, C. T. H. Davies, G. C. Donald, J. Koponen, and G. P. Lepage (HPQCD Collaboration), Nonperturbative comparison of clover and highly improved staggered quarks in lattice QCD and the properties of the $\phi$ meson, Phys. Rev. D 96, 074502 (2017).

[73] B. Bhattacharya, A. Datta, J.-P. Guevin, D. London, and R. Watanabe, Simultaneous explanation of the $R_{K}$ and $R_{D^{(*)}}$ puzzles: A model analysis, J. High Energy Phys. 01 (2017) 015.

[74] G. Valencia, Long distance contribution to $K_{L} \rightarrow \ell^{+} \ell^{-}$, Nucl. Phys. B517, 339 (1998).

[75] G. Isidori and R. Unterdorfer, On the short distance constraints from $K_{L, S} \rightarrow \mu^{+} \mu^{-}$, J. High Energy Phys. 01 (2004) 009.
[76] V. Cirigliano and I. Rosell, $\pi / K \rightarrow e \bar{\nu}_{e}$ branching ratios to $O\left(e^{2} p^{4}\right)$ in chiral perturbation theory, J. High Energy Phys. 10 (2007) 005.

[77] S. Aoki, G. Cossu, X. Feng, H. Fukaya, S. Hashimoto, T. Kaneko, J. Noaki, and T. Onogi (JLQCD Collaboration), Chiral behavior of $K \rightarrow \pi l \nu$ decay form factors in lattice QCD with exact chiral symmetry, Phys. Rev. D 96, 034501 (2017).

[78] J. M. Flynn, T. Izubuchi, T. Kawanai, C. Lehner, A. Soni, R. $\mathrm{S}$. Van de Water, and O. Witzel, $B \rightarrow \pi \ell \nu$ and $B_{s} \rightarrow K \ell \nu$ form factors and $\left|V_{u b}\right|$ from $2+1$-flavor lattice QCD with domain-wall light quarks and relativistic heavy quarks, Phys. Rev. D 91, 074510 (2015).

[79] A. M. Baldini et al. (MEG Collaboration), Search for the lepton flavour violating decay $\mu^{+} \rightarrow e^{+} \gamma$ with the full dataset of the MEG experiment, Eur. Phys. J. C 76, 434 (2016).

[80] B. Aubert et al. (BABAR Collaboration), Searches for Lepton Flavor Violation in the Decays $\tau^{ \pm} \rightarrow e^{ \pm} \gamma$ and $\tau^{ \pm} \rightarrow \mu^{ \pm} \gamma$, Phys. Rev. Lett. 104, 021802 (2010).

[81] R. Kitano, M. Koike, and Y. Okada, Detailed calculation of lepton flavor violating muon electron conversion rate for various nuclei, Phys. Rev. D 66, 096002 (2002); Erratum, Phys. Rev. D76, 059902(E) (2007).

[82] V. Cirigliano, R. Kitano, Y. Okada, and P. Tuzon, On the model discriminating power of $\mu \rightarrow e$ conversion in nuclei, Phys. Rev. D 80, 013002 (2009).

[83] T. S. Kosmas, S. Kovalenko, and I. Schmidt, Nuclear $\mu^{-}-e^{-}$conversion in strange quark sea, Phys. Lett. B 511, 203 (2001).

[84] T. Suzuki, D. F. Measday, and J. P. Roalsvig, Total nuclear capture rates for negative muons, Phys. Rev. C 35, 2212 (1987). 